\title{
The route to dissipation in strongly stratified and rotating flows
}

\author{
Enrico Deusebio $\dagger$, A. Vallgren and E. Lindborg \\ Linné Flow Centre, Department of Mechanics, Royal Institute of Technology, Stockholm, 10044, Sweden \\ (Received 20 February 2012; revised 4 December 2012; accepted 6 December 2012; \\ first published online 27 February 2013)
}

We investigate the route to dissipation in strongly stratified and rotating systems through high-resolution numerical simulations of the Boussinesq equations (BQs) and the primitive equations (PEs) in a triply periodic domain forced at large scales. By applying geostrophic scaling to the BQs and using the same horizontal length scale in defining the Rossby and the Froude numbers, $R o$ and $F r$, we show that the PEs can be obtained from the BQs by taking the limit $F r^{2} / R o^{2} \rightarrow 0$. When $F r^{2} / R o^{2}$ is small the difference between the results from the BQ and the PE simulations is shown to be small. For large rotation rates, quasi-geostrophic dynamics are recovered with a forward enstrophy cascade and an inverse energy cascade. As the rotation rate is reduced, a fraction of the energy starts to cascade towards smaller scales, leading to a shallowing of the horizontal spectra from $k_{h}^{-3}$ to $k_{h}^{-5 / 3}$ at the small-scale end. The vertical spectra show a similar transition as the horizontal spectra and we find that Charney isotropy is approximately valid also at larger wavenumbers than the transition wavenumber. The high resolutions employed allow us to capture both ranges within the same simulation. At the transition scale, kinetic energy in the rotational and in the horizontally divergent modes attain comparable values. The divergent energy is several orders of magnitude larger than the quasi-geostrophic divergent energy given by the $\Omega$-equation. The amount of energy cascading downscale is mainly controlled by the rotation rate, with a weaker dependence on the stratification. A larger degree of stratification favours a downscale energy cascade. For intermediate degrees of rotation and stratification, a constant energy flux and a constant enstrophy flux coexist within the same range of scales. In this range, the enstrophy flux is a result of triad interactions involving three geostrophic modes, while the energy flux is a result of triad interactions involving at least one ageostrophic mode, with a dominant contribution from interactions involving two ageostrophic and one geostrophic mode. Dividing the ageostrophic motions into two classes depending on the sign of the linear wave frequency, we show that the energy transfer is for the largest part supported by interactions within the same class, ruling out the wave-wave-vortex resonant triad interaction as a mean of the downscale energy transfer. The role of inertia-gravity waves is studied through analyses of time-frequency spectra of single Fourier modes. At large scales, distinct peaks at frequencies predicted for linear waves are observed, whereas at small scales no clear wave activity is observed. Triad interactions show a behaviour which is consistent with turbulent dynamics, with a large exchange of 
energy in triads with one small and two large comparable wavenumbers. The exchange of energy is mainly between the modes with two comparable wavenumbers.

Key words: geostrophic turbulence, rotating turbulence, stratified turbulence

\section{Introduction}

Flows in the atmosphere and in the oceans develop over an extremely wide range of scales, both in time and space. The atmosphere is largely forced at scales of the order of $1000 \mathrm{~km}$, where baroclinic instability converts available potential energy, related to the meridional temperature gradient, to kinetic energy. Similarly, the general circulation of the oceans is mainly driven by surface fluxes of momentum at scales as large as $1000 \mathrm{~km}$. On the other hand, the dissipation of energy can only be achieved by molecular friction and diffusion. As opposed to the large-scale forcing, viscosity and diffusivity act at very small scales, which can be estimated to be of the order of centimetres. How energy can cascade from the largest to the smallest scales, over a range of about eight orders of magnitude, is not fully understood (Muller, McWilliams \& Molemaker 2005). Even though the nonlinearities in the Navier-Stokes (NS) equations provide a mechanism for energy transfer between scales, the routes to dissipation are presently not clear. The general problem of how energy can be transferred from the very largest to the very smallest scales in geophysical flows has recently been the subject of several studies (Muller et al. 2005; Waite \& Bartello 2006; Molemaker, McWilliams \& Capet 2010).

At synoptic scales, of the order of $1000 \mathrm{~km}$, atmospheric dynamics are highly affected by both rotation and stratification. The relative importance of Coriolis forces and buoyancy forces as compared with inertial forces are often quantified by the Rossby and the Froude numbers, defined as

$$
R o=\frac{U}{f l}, \quad F r=\frac{U}{N l} .
$$

Here, $U$ is a characteristic velocity, $l$ a characteristic length, $f=2 \Omega \sin \theta$ is the Coriolis parameter, with $\Omega$ being the rotation rate and $\theta$ the latitude, and $N$ is the Brunt-Väisälä frequency. Note that we have not used a vertical length scale in the definition of $F r$, as is common practise, but rather the same horizontal length scale $l$ as in the definition of $R o$. With these definitions we have $F r / R o=f / N$. In the limit of very strong rotation and stratification, the NS equations can be reduced to the so-called quasi-geostrophic $(\mathrm{QG})$ equation, stating that the potential vorticity, $q$, is materially conserved (Charney 1971). Therefore, the QG equation conserves independently two quadratic invariants: total energy, which is the sum of potential and kinetic energy, and potential enstrophy, defined as $q^{2} / 2$. In this limit there is an inverse cascade of energy dominating the large scales and a forward cascade of enstrophy dominating the small scales, just as in two-dimensional turbulent flows (Kraichnan 1967). The energy spectrum scales as $k^{-5 / 3}$ in the energy inertial range and as $k^{-3}$ in the enstrophy inertial range. Recent high-resolution numerical simulations (Scott 2007; Boffetta \& Musacchio 2010; Vallgren \& Lindborg 2011) have mainly confirmed this picture, although some anomalous effects due to large-scale vortices have also been reported (Scott 2007; Vallgren 2011). 
Cambon, Mansour \& Godeferd (1997) studied the effect of system rotation on the downscale energy transfer. As the rotation rate is increased, the energy cascade is inhibited and the forward energy transfer terms are damped, leading to a reduction of the small-scale dissipation. Indeed, the inverse-cascade dynamic of strongly rotating and stratified systems seems to be inconsistent with small-scale energy dissipation, posing the intriguing question of how and where the transfer might take place (Wunsch \& Ferrari 2004; Muller et al. 2005; Ferrari \& Wunsch 2009). Interaction between preexisting inertia gravity waves and large mesoscale motions may provide a possible mechanism (Nikurashin \& Ferrari 2011). Indeed, also boundary effects as top and bottom turbulent boundary layer and internal waves produced by topography can lead to small-scale dissipation (Ferrari \& Wunsch 2009). This route may be important in the oceans where most of the energy injected by the surface wind stresses is dissipated close to the sea surface. However, there is also 'a direct route to dissipation in the ocean interior by a forward energy cascade' (Molemaker et al. 2010). An interesting perspective is offered by the hypothesis that finite rotation rates lead to a transfer of energy from balanced, geostrophic motions to unbalanced motions (Bartello 1995; Waite \& Bartello 2006; Bartello 2010; McWilliams 2010; Molemaker et al. 2010). Possible mechanisms underlying this transfer may be frontogenetic and frontal instabilities, mixed-layer instabilities, localized diapycnal mixing and surface filamentogenesis (McWilliams 2010). The QG equation is not able to capture this process. Therefore, the primitive equations (PEs) or the Boussinesq equations (BQs) should rather be considered. The BQs with hydrostasy assumed in the vertical direction is often referred as PEs. Stability analysis of a non-geostrophic and nonhydrostatic Eady problem shows the appearance of new kind of instabilities apart from the classical baroclinic instability. Such instability may lead to a transfer of energy from geostrophic to ageostrophic motions (Molemaker, McWilliams \& Yavneh 2005). Viúdez \& Dritschel (2006) studied the breakdown of a baroclinic geostrophic jet, finding the emission of unbalanced wave motions with frequencies close to the inertial frequency. Moreover, it has recently been shown that forced Eady flows can relax to statistically stationary states only if ageostrophic motions are taken into account (Molemaker \& McWilliams 2010). That is to say, QG flows cannot establish efficient and steady routes to dissipation.

Numerical simulations of strongly stratified flows (Riley \& deBruynKops 2003; Waite \& Bartello 2004; Lindborg 2006; Brethouwer et al. 2007) have shown that a forward energy cascade can develop and that a steady route to dissipations can be maintained, also in weakly rotating systems (Lindborg 2006; Waite \& Bartello 2006). Using an eddy-damped quasinormal Markovian (EDQNM) closure, Godeferd \& Cambon (1994) argued that a stable stratification may create a strongly anisotropic structure which prevents the development of an inverse cascade of energy. As already noted by Lilly (1983), stratification results in a decoupling of the dynamics into layers, leading to large gradients in the vertical direction. The resulting Kelvin-Helmholtz $(\mathrm{KH})$ instabilities provide a mechanism for a downscale energy cascade. Whereas the horizontal Froude number, $F r=U / N l$, is very small, $\mathrm{KH}$ instabilities keep the vertical Froude number, $F r_{v}=U / N h$, of the order of unity (Billant \& Chomaz 2001; Riley \& deBruynKops 2003), naturally setting the flow layers thickness. Here, $l$ and $h$ are the characteristic horizontal and vertical length scale, respectively. The ratio $\alpha=h / l=F r / F r_{v}$ is a very small quantity in strongly stratified flows, which means that flow structures are highly elongated in the horizontal direction and very confined in the vertical. Such structures are often referred to as pancake structures. Waite \& Bartello (2006) studied the vertical length scales for stratified and rotating geostrophic 
turbulence. For small Ro, they found that the scaling suggested by Charney (1971), $h \sim l f / N$, applies. On the other hand, for $R o>0.1, F r_{v}$ became of the order of unity and independent of $R o$ and $F r$, in agreement with the prediction of Billant \& Chomaz (2001) for stratified turbulence. As in three-dimensional turbulence, both kinetic and potential horizontal wavenumber energy spectra of strongly stratified flows scale as $k_{h}^{-5 / 3}$. Lindborg \& Brethouwer (2007) showed that in this range, rotational and divergent modes, often referred to as wave and vortical modes, have comparable magnitude. In contrast to what has been suggested in many studies (e.g. Lelong \& Riley 1991), they showed that there is no strict dynamic decoupling between these two types of modes. The reason for this is that they develop on comparable time scales, as suggested by the analysis of Billant \& Chomaz (2001).

Observations in the oceans and in the atmosphere support the hypothesis that there is a downscale energy cascade over a wide range of scales. In the oceans, Ménesguen et al. (2009) studied the structure of long-lived anticyclonic lens-shaped vortices known as Meddies through fine-resolution geoseismic sections and highresolution numerical simulations. They were able to demonstrate the presence of a downscale energy cascade over roughly one decade, extending up to scales of the order of $3 \mathrm{~km}$. In the atmosphere, wind and potential temperature spectra calculated from aircraft measurements (Nastrom \& Gage 1985; Cho \& Lindborg 2001) show two distinct range of scales. At synoptic scales, between 500 and $1000 \mathrm{~km}$, a $k_{h}^{-3}$ spectrum is found, consistent with a two-dimensional-like turbulent dynamics within the enstrophy range. In the mesoscale range, below $500 \mathrm{~km}$, the spectra shallow significantly, attaining scaling exponents close to $-5 / 3$. Third-order structure function analysis has revealed that there is a downscale energy flux in this range (Cho \& Lindborg 2001).

The transition from a $k_{h}^{-3}$ to a $k_{h}^{-5 / 3}$ spectrum have been simulated both in idealized numerical simulations (Kitamura \& Matsuda 2006; Bartello 2010; Vallgren, Deusebio \& Lindborg 2011) and atmospheric models (Skamarock 2004; Takahashi, Hamilton \& Ohfuchi 2006; Hamilton, Takahashi \& Ohfuchi 2008; Waite \& Snyder 2009). Bartello (2010) simulated a strongly stratified and rotating flows in a triply periodic domain forced only at large scales. Vallgren et al. (2011) considered instead the PEs and showed that the ratio between the energy going downscale and upscale increases with $R o$ and small-scale dissipation scales as $R o^{3 / 2}$. The increasing amount of energy going downscale as $R o$ was increased led to a shallowing of the energy spectra to $k_{h}^{-5 / 3}$ in both studies, consistent with observations.

At the present point, it is not entirely clear whether the forward energy cascade is a result of stratified turbulence or resonant interacting waves. Several theories based on the assumption that waves play a central role in the route to dissipation have been proposed. In the atmosphere, Waite \& Snyder (2009) simulated an idealized baroclinic wave life cycle, finding a shallowing of the kinetic energy spectra due to the divergent contributions. The authors argued that waves spontaneously emitted in the dynamics propagate vertically and lead to the shallowing of the energy spectra. In the ocean, spectra show remarkable similarities from place to place, as described by Garrett \& Munk (1979), who proposed a model (later improved by Munk (1981)) for describing the frequency and vertical wavenumber spectra (hereafter referred to as GM spectra). A $k_{z}^{-2}$ spectrum down to scales of roughly $10 \mathrm{~m}$ is predicted and is usually interpreted as the result of the superposition of saturated waves. At smaller scales, the GM spectrum is followed by a steepening to $k_{z}^{-3}$ (Gargett et al. 1981). This transition is assumed to be set by the onset of wave instabilities, i.e. waves reaching the critical 
steepness $u_{r m s} / c_{x}>1$, where $u_{r m s}$ is the velocity fluctuation and $c_{x}$ is the phase velocity of the wave, such that overturning and breaking processes are allowed. Nevertheless, it must be noted that vertical spectra of the form $N^{2} k_{z}^{-3}$ is also predicted by theories of stratified turbulence (Billant \& Chomaz 2001). Such a prediction has recently been confirmed by direct numerical simulations (Augier, Chomaz \& Billant 2012) of strongly stratified flows at scales larger than the Ozmidov length scale without the presence of any clear strong wave signals. That stratified turbulence may also be an important dynamical process in the oceans is supported by the observations and the simulations of Ménesguen et al. (2009).

The nonlinear terms in the NS equations allow energy to be transferred among the different modes, involving triads in spectral space. Through resonant interaction, waves can support an energy transfer towards small scales without involving any turbulentlike motions (Bellet et al. 2006; Polzin \& Lvov 2011). Phillips (1981) and Staquet \& Sommeria (2002) reviewed the condition and the mechanism for subharmonic parametric instability (SPI) for which a long wave $\left(k_{0}\right)$ resonantly interacts with two high wavenumber secondary waves $\left(k_{1}, k_{2} \gg k_{0}\right)$ which have half the frequency with respect to the primary wave $\left(\omega_{1}=\omega_{2}=-\omega_{0} / 2\right)$. The transfer of energy is mainly directed from the long wave to the short waves, feeding their growth. The resonant scatter of energy between two high-wavenumber high-frequency waves due to a largescale low-frequency wave is often referred to as induced diffusion (ID) (McComas \& Bretherton 1977; Staquet \& Sommeria 2002; Polzin \& Lvov 2011). McComas \& Bretherton (1977) have interpreted the GM spectrum as the result of weakly interacting resonant waves. However, Lvov, Polzin \& Yokoyama (2013) warn about the possibility that one of the assumptions, namely the stationarity of the GM spectrum with respect to the resonant interactions, may not be satisfied and that near-resonant interactions must be taken into account. Resonance could possibly occur also between two waves with similar frequency and a vortical mode with zero frequency (Lelong \& Riley 1991; Bartello 1995). As predicted by statistical equilibrium analysis, energy is expected to flow from large-scale to small-scale inertia-gravity waves. According to the analysis of Bartello (1995), resonance interactions between three waves are of secondary importance. Ageostrophic energy can instead cascade downscale thanks to the wave-wave-vortex interactions.

Within the context of turbulence theory, triad interactions have historically been the subject of a great number of modelling efforts (see for instance Leith \& Kraichnan 1972). Ohkitani \& Kida (1992) carried out the first detailed numerical study of triad interactions in forced isotropic turbulence. Similar analyses were also carried out in the context of two-dimensional turbulence, covering both the enstrophy and the energy ranges (Ohkitani 1990; Maltrud \& Vallis 1993; Vallgren 2011). The main question addressed in these studies is whether the transfer of energy is local in wavenumber space. Somewhat surprisingly, all of the aforementioned studies showed that turbulence is an intimately non-local process, involving large exchange of energy in triads with two large comparable wavenumbers and one small wavenumber. Despite the high non-locality, energy exchange is mainly between the two large wavenumbers, whereas the small one only acts as a catalyser.

In this paper, we will mainly address two issues. First, we aim at extending some recent results by Vallgren et al. (2011), obtained within the framework of the PEs, by considering the full BQs where no hydrostatic balance is assumed in the vertical direction. The main focus is to understand whether and how the route to dissipation is modified by a non-zero horizontal Froude number. Comparisons with the PEs are made, mainly focusing on energy and enstrophy spectra and fluxes. 
The simulations we have carried out give a good picture of the dynamics which take place for several $R o$ and $F r$, spanning reasonable values for large-scale atmospheric flows. Second, we study the possible influence of wave motions in the forward energy cascade. To do this, the BQs is the appropriate set of equations. In fact, the PEs do not correctly represent wave modes that have a long vertical and a short horizontal wavelength, which have fast frequencies and for which the hydrostatic approximation holds to a smaller degree. Nevertheless, as we will show in the following, the dynamics appear to be very similar in the two cases, indicating that these modes may be of minor importance in the overall dynamics. Frequency analyses of time series from both geostrophically balanced modes and ageostrophic modes are carried out in order to find signatures of wave motions. We also study triad interactions in order to understand which modes contribute the most to the energy transfer towards small scales and whether resonant wave interactions or turbulent-like process are dominant.

The paper is organized as follows: $\S 2$ gives the theoretical background and the formulation of the problem; in $\S 3$ the numerical details and parameters are summarized; in $\S 4$ we present some flow fields in physical space; in $\S 5$, energy spectra and fluxes are presented with comparisons between the PEs and the BQs; $\S 6$ focuses on analysing wave motions and frequency spectra; in $\S 7$ the transfer of energy among wavenumbers through triad interactions is studied. Conclusions are finally given in $\S 8$.

\section{Theoretical formulation}

We start from the inviscid three-dimensional NS equations within the Boussinesq approximation in a rotating frame for an incompressible flow,

$$
\begin{aligned}
\frac{\mathrm{D} \boldsymbol{u}_{h}}{\mathrm{D} t} & =-\frac{\nabla_{h} p}{\rho_{0}}-f \boldsymbol{e}_{z} \times \boldsymbol{u}_{h}, \\
\frac{\mathrm{D} w}{\mathrm{D} t} & =-\frac{\partial p}{\partial z}+b, \\
\frac{\mathrm{D} b}{\mathrm{D} t} & =-N^{2} w, \\
\nabla \cdot \boldsymbol{u} & =0,
\end{aligned}
$$

where $\delta_{i 3}$ is the Kronecker's delta, $\boldsymbol{u}$ is the velocity vector, the subscript $h$ indicates the horizonal $(x-y)$ component, $w$ is the vertical velocity component, $\boldsymbol{e}_{z}$ is the vertical unit vector, $p$ is the pressure, $b=g \rho /\left(\rho_{0}\right)$ is the buoyancy, where $\rho$ and $\rho_{0}$ are the fluctuating and background densities, respectively. In atmospheric and oceanographic applications, potential density is used in place of $\rho$ (Vallis 2006).

The system (2.1) can be rewritten introducing the Charney potential vorticity $q$ and the two ageostrophic components, $a_{1}$ and $a_{2}$ :

$$
\begin{aligned}
q & =-\frac{\partial u}{\partial y}+\frac{\partial v}{\partial x}+\frac{f}{N^{2}} \frac{\partial b}{\partial z}, \\
a_{1} & =-\frac{f}{N} \frac{\partial v}{\partial z}+\frac{1}{N} \frac{\partial b}{\partial x}, \\
a_{2} & =\frac{f}{N} \frac{\partial u}{\partial z}+\frac{1}{N} \frac{\partial b}{\partial y},
\end{aligned}
$$


where $a_{1}$ and $a_{2}$ measure the departure from the thermal wind balance (Vallis 2006). With periodic boundary conditions in the horizontal and rigid lid or no-slip boundary conditions in the vertical, the whole flow field can be inverted from $q, a_{1}$ and $a_{2}$. However, if periodic boundary conditions are applied also in the vertical, the barotropic modes of the vertical velocity, that are Fourier modes for which the vertical wavenumber is zero, need to be solved explicitly. Note that the decomposition of the flow in a geostrophic and ageostrophic parts is similar but not equivalent to that used by Gill (1982) and Viúdez \& Dritschel (2006). It may be shown (appendix B) that the decomposition in $q, a_{1}, a_{2}$ and the normal modes (Bartello 1995) are related by a linear transformation. Even though some important properties of the Charney potential vorticity are lost within the $\mathrm{BQs}$, e.g. its material conservation, we nevertheless retain its use rather than considering other nonlinear (but conserved) quantities such as the Ertel potential vorticity. In contrast, the Charney potential vorticity is linear in $u, v$ and $b$, therefore making the potential enstrophy $q^{2} / 2$ quadratic when expressed in basic flow quantities. In the following, when not otherwise stated, we will refer to the Charney potential vorticity simply as 'potential vorticity' $q$.

We make system (2.1) and (2.2) dimensionless by introducing the following definitions

$$
\begin{gathered}
\hat{x}=x / l, \quad \hat{y}=y / l, \quad \hat{z}=z / h, \quad \hat{t}=t U / l, \\
\hat{u}=u / U, \quad \hat{v}=v / U, \quad \hat{w}=w N l / U^{2}, \quad \hat{b}=b / N U, \quad \hat{p}=p / f U l .
\end{gathered}
$$

Note that two different length scales are used in the horizontal and in the vertical direction, $l$ and $h$, respectively. The estimate for the pressure follows from the fact that, for flows close to geostrophy, the horizontal pressure gradient is mainly balanced by the Coriolis force. The scaling for the buoyancy $b$ follows from the assumption that the potential energy is of the same order of magnitude as the kinetic energy. Indeed, this has been confirmed by a number of studies in QG (Vallgren \& Lindborg 2010) and stratified (Lindborg 2006; Brethouwer et al. 2007; Lindborg \& Brethouwer 2007) turbulence. From $U, f, N, l$ and $h$, three independent dimensionless groups can be formed: the Rossby number Ro, the Froude number $F r$ and the Burger number $B u=N h / f$. The dimensionless counterparts of $q, a_{1}$ and $a_{2}$ are

$$
\hat{q}=q l / U, \quad \hat{a}_{1}=a_{1} l / U R o, \quad \hat{a}_{2}=a_{2} l / U R o .
$$

In order to simplify the notation, we will from now and on drop the hats when referring to dimensionless quantities. Using the definitions (2.2), we recast (2.1) into the three prognostic dimensionless equations

$$
\begin{aligned}
\frac{\partial q}{\partial t}= & \frac{\partial}{\partial y}\left(\frac{\partial u^{2}}{\partial x}+\frac{\partial u v}{\partial y}+\frac{R o}{B u} \frac{\partial u w}{\partial z}\right)-\frac{\partial}{\partial x}\left(\frac{\partial u v}{\partial x}+\frac{\partial v^{2}}{\partial y}+\frac{R o}{B u} \frac{\partial v w}{\partial z}\right) \\
& -\frac{1}{B u} \frac{\partial}{\partial z}\left(\frac{\partial u b}{\partial x}+\frac{\partial v b}{\partial y}+\frac{R o}{B u} \frac{\partial w b}{\partial z}\right)+v_{S} \nabla^{8} q-v_{L} q,
\end{aligned}
$$




$$
\begin{aligned}
R o \frac{\partial}{\partial t}\left(a_{1}+\frac{F r^{2}}{R o^{2}} \frac{\partial w}{\partial y}\right)= & a_{2}-\frac{\partial w}{\partial x}+\frac{1}{B u} \frac{\partial}{\partial z}\left(\frac{\partial u v}{\partial x}+\frac{\partial v^{2}}{\partial y}+\frac{R o}{B u} \frac{\partial v w}{\partial z}\right) \\
& -\frac{\partial}{\partial x}\left(\frac{\partial u b}{\partial x}+\frac{\partial v b}{\partial y}+\frac{R o}{B u} \frac{\partial w b}{\partial z}\right) \\
& -\frac{F r^{2}}{R o} \frac{\partial}{\partial y}\left(\frac{\partial u w}{\partial x}+\frac{\partial v w}{\partial y}+\frac{R o}{B u} \frac{\partial w w}{\partial z}\right) \\
& +\operatorname{Rov}_{S} \nabla^{8} a_{1}-\operatorname{Rov}_{L} a_{1}, \\
R o \frac{\partial}{\partial t}\left(a_{2}-\frac{F r^{2}}{R o^{2}} \frac{\partial w}{\partial x}\right)= & -a_{1}-\frac{\partial w}{\partial y}-\frac{1}{B u} \frac{\partial}{\partial z}\left(\frac{\partial u^{2}}{\partial x}+\frac{\partial u v}{\partial y}+\frac{R o}{B u} \frac{\partial u w}{\partial z}\right) \\
& -\frac{\partial}{\partial y}\left(\frac{\partial u b}{\partial x}+\frac{\partial v b}{\partial y}+\frac{R o}{B u} \frac{\partial w b}{\partial z}\right) \\
& +\frac{F r^{2}}{R o} \frac{\partial}{\partial x}\left(\frac{\partial u w}{\partial x}+\frac{\partial v w}{\partial y}+\frac{R o}{B u} \frac{\partial w w}{\partial z}\right) \\
& +R_{o v} \nabla^{8} a_{2}-R o v_{L} a_{2} .
\end{aligned}
$$

The buoyancy and the velocity components can be calculated from $q, a_{1}$ and $a_{2}$ using the relations

$$
\begin{gathered}
\left(\frac{\partial^{2}}{\partial x^{2}}+\frac{\partial^{2}}{\partial y^{2}}+\frac{1}{B u^{2}} \frac{\partial^{2}}{\partial z^{2}}\right) b=\frac{1}{B u} \frac{\partial q}{\partial z}+R o\left(\frac{\partial a_{1}}{\partial x}+\frac{\partial a_{2}}{\partial y}\right) \\
\frac{1}{B u} \frac{\partial u}{\partial z}=-\frac{\partial b}{\partial y}+R o a_{2} \\
\frac{1}{B u} \frac{\partial v}{\partial z}=\frac{\partial b}{\partial x}-R o a_{1} \\
\frac{1}{B u^{2}} \frac{\partial^{2} w}{\partial^{2} z}=\frac{\partial a_{1}}{\partial y}-\frac{\partial a_{2}}{\partial x}
\end{gathered}
$$

The system (2.5)-(2.6) constitutes a complete closed set which contains the three dimensionless parameters $R o, F r$ and $B u$. However, we note that $B u$ and $z$ always appear together. If the characteristic aspect ratio, $\alpha=h / l$, is not determined by initial or boundary conditions, but rather by the dynamics itself, then it may be argued that $B u=\alpha R o / F r \sim 1$ in the limit of small $R o$ and $F r$, since a maximum number of terms will stay of leading order in the system (2.5). This is a similar argument as developed by Billant \& Chomaz (2001) who found that $\alpha \sim F r$ in strongly stratified turbulence. That $B u \sim 1$ in the strongly stratified and rotating regime was already argued by Charney (1971). Thus, in the following we assume $B u=1$ in the system (2.5)-(2.6). Both a small-scale hyperviscosity, associated with a fourth-order Laplacian, and large-scale linear drag have been employed in order to provide a mean to dissipate energy at small and large scales. The use of hyperviscosity allows us to reduce the range of small scales at which viscosity plays a dominant role. Note that the dissipation operator is isotropic in the space where the vertical coordinate has been stretched. This means that it is anisotropic in the real space where the vertical coordinate is unstretched. Large-scale friction is needed in order to avoid that an energy condensate develops at large scales as a result of the inverse energy cascade. Note that for simplicity, the set of (2.5) has been derived assuming that there is a 
large-scale and a small-scale diffusivity associated with the buoyancy which is equal to the corresponding large-scale and small-scale viscosity $v_{L}$ and $v_{S}$, respectively.

Taking the scalar product of (2.1a) and $\boldsymbol{u}$, multiplying (2.1c) by $b$, and summing the resulting equations, we can derive the total energy equation in a periodic frame

$$
\frac{\mathrm{d}}{\mathrm{d} t} \int \frac{u^{2}+v^{2}+F r^{2} w^{2}+b^{2}}{2} \mathrm{~d} x \mathrm{~d} y \mathrm{~d} z=-\varepsilon,
$$

where $\varepsilon$ is the sum of the small-scale and large-scale energy dissipation, $\varepsilon_{S}$ and $\varepsilon_{L}$, respectively. The total energy of the system is the sum of kinetic energy, $\left(u^{2}+v^{2}+F r^{2} w^{2}\right) / 2$, and potential energy, $b^{2} / 2$.

With $R o \sim 1$, the geostrophically scaled version of the PEs is obtained by taking the limit $F r^{2} \rightarrow 0$ in $(2.5 a)-(2.5 c)$. PEs is the set of equations that is obtained by assuming hydrostatic balance in $(2.1 b)$ and the condition $F r^{2}=F r_{v}^{2}(h / l)^{2} \ll 1$ is the same condition for hydrostatic balance as is given in textbooks on geophysical fluid dynamics (e.g. Vallis 2006). However, with a small but finite Rossby number, the condition for neglecting the second term on the right-hand side of $(2.5 b)$ and $(2.5 c)$, while keeping the first term, is $(F r / R o)^{2} \ll 1$, which is a stronger condition.

Interestingly, the potential vorticity (2.5a) does not depend on $\mathrm{Fr}$ and therefore has the same expression both in the PEs and in the BQs. Neglecting the viscous terms and using the definitions (2.2) and (2.5a) can be rewritten as

$$
\begin{aligned}
\frac{\mathrm{D} q}{\mathrm{D} t}= & R o\left(a_{1} \frac{\partial b}{\partial y}-a_{2} \frac{\partial b}{\partial x}+q \frac{\partial w}{\partial z}-\frac{\partial b}{\partial y} \frac{\partial w}{\partial y}-\frac{\partial b}{\partial x} \frac{\partial w}{\partial x}-2 \frac{\partial b}{\partial z} \frac{\partial w}{\partial z}\right) \\
& +R o^{2}\left(a_{1} \frac{\partial w}{\partial x}+a_{2} \frac{\partial w}{\partial y}\right) .
\end{aligned}
$$

Note that whereas the Ertel potential vorticity (e.g. Pedlosky 1987) is a conserved quantity also in PEs and BQs, the same does not apply to the Charney potential vorticity which we consider here. However, in the limit $R o \rightarrow 0$, the above expression reduces to

$$
\frac{\mathrm{D} q}{\mathrm{D} t}=0,
$$

stating that $q$ is a materially conserved quantity (Charney 1971). In the limit $R o \rightarrow 0$ and $F r^{2} / R o^{2} \rightarrow 0$, the (2.5b) and (2.5c) lose their time derivative and reduce to algebraic equations from which the ageostrophic wind may be directly computed from geostrophically balanced fields (see e.g. Gill 1982).

Retaining only the linear terms in $(2.5 b)$ and $(2.5 c)$ and using $(2.1 d)$, we can derive the well-known wave equation for inertia-gravity waves (Gill 1982; Pedlosky 1987; Vallis 2006). The corresponding dispersion relation reads

$$
\omega_{d}^{2}=\frac{k_{x}^{2}+k_{y}^{2}+k_{z}^{2}}{F r^{2}\left(k_{x}^{2}+k_{y}^{2}\right)+R o^{2} k_{z}^{2}} .
$$

Note that the dimensional counterpart of (2.10) would read

$$
\tilde{\omega}_{d}^{2}=\frac{N^{2}\left(\tilde{k}_{x}^{2}+\tilde{k}_{y}^{2}\right)+f^{2} \tilde{k}_{z}^{2}}{\tilde{k}_{x}^{2}+\tilde{k}_{y}^{2}+\tilde{k}_{z}^{2}},
$$


which is the common expression which may be found in any geophysical fluid

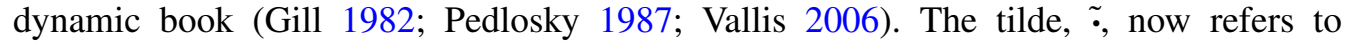
dimensional quantities.

In the limit of zero $F r$, the dispersion relation (2.10) becomes singular for small $k_{z}$, i.e. for vertically long, barotropic waves. Infinitely fast wave motions may therefore appear in the PEs. On the other hand, in the BQs wave frequencies are bounded between $\mathrm{Ro}^{-1}$ and $\mathrm{Fr}^{-1}$, as is clear from (2.10).

The total energy spectrum

$$
E(\boldsymbol{k})=\frac{\hat{u}(\boldsymbol{k}) \hat{u}^{*}(\boldsymbol{k})+\hat{v}(\boldsymbol{k}) \hat{v}^{*}(\boldsymbol{k})+F r^{2} \hat{w}(\boldsymbol{k}) \hat{w}^{*}(\boldsymbol{k})+\hat{b}(\boldsymbol{k}) \hat{b}^{*}(\boldsymbol{k})}{2},
$$

decouples into a geostrophic part associated with $q$ and an ageostrophic part associated with $a_{1}$ and $a_{2}$. We find that

$$
E(\boldsymbol{k})=\sum_{\boldsymbol{k}} \frac{\hat{q}(\boldsymbol{k}) \hat{q}^{*}(\boldsymbol{k})}{2 k^{2}}+\left\{\begin{array}{l}
\hat{a}_{1}(\boldsymbol{k}) \\
\hat{a}_{2}(\boldsymbol{k})
\end{array}\right\}\left[\begin{array}{ll}
E_{22} & E_{23} \\
E_{23} & E_{33}
\end{array}\right]\left\{\begin{array}{c}
\hat{a}_{1}(\boldsymbol{k}) \\
\hat{a}_{2}(\boldsymbol{k})
\end{array}\right\}^{*},
$$

where $k=\sqrt{k_{x}^{2}+k_{y}^{2}+k_{z}^{2}}$. The decomposition (2.13) is valid for baroclinic modes. For barotropic modes the energy spectrum can be divided into a part which is associated with $q$ and is equal to the contribution from the two horizontal velocity components and a part which is equal to the contribution from $w$ and $b$. The total energy can therefore be divided into a geostrophic and an ageostrophic part, $E_{G}$ and $E_{A}$, respectively. In the appendix $\mathrm{A}$, the explicit form of the matrix $\boldsymbol{E}$ is given. Following Bartello (1995), we will now classify the nonlinear interactions involving geostrophic and ageostrophic modes. According to (2.13), the rate of change of the geostrophic energy, $E_{G}$, can be rewritten as

$$
\frac{\partial E_{G}}{\partial t}=\sum_{\boldsymbol{k}} \frac{1}{2 k^{2}}\left(\frac{\partial \hat{q}(\boldsymbol{k})}{\partial t} \hat{q}^{*}(\boldsymbol{k})+\frac{\partial \hat{q}^{*}(\boldsymbol{k})}{\partial t} \hat{q}(\boldsymbol{k})\right)
$$

where the rate of change of $q$ due to nonlinear terms can be separated into three contributions

$$
\frac{\partial \hat{q}(\boldsymbol{k})}{\partial t}=N L_{G G}(\boldsymbol{k})+N L_{G A}(\boldsymbol{k})+N L_{A A}(\boldsymbol{k})
$$

with the subscripts standing for the nonlinear terms arising from the interaction of the two classes of motions: geostrophic $(\mathrm{G})$ and ageostrophic (A). Explicitly,

$$
\begin{gathered}
N L_{G G}(\boldsymbol{k})=\sum_{\substack{\boldsymbol{p}, \boldsymbol{q} \\
k=\boldsymbol{p}+\boldsymbol{q}}} \Gamma_{G G}(\boldsymbol{k}, \boldsymbol{p}, \boldsymbol{q}) \hat{q}(\boldsymbol{p}) \hat{q}(\boldsymbol{q}), \\
N L_{G A}(\boldsymbol{k})=\sum_{\substack{\boldsymbol{p}, \boldsymbol{q} \\
k=p+q}} \sum_{i} \Gamma_{G A_{i}}(\boldsymbol{k}, \boldsymbol{p}, \boldsymbol{q})\left[\hat{q}(\boldsymbol{p}) \hat{a}_{i}(\boldsymbol{q})+\hat{a}_{i}(\boldsymbol{p}) \hat{q}(\boldsymbol{q})\right], \\
N L_{A A}(\boldsymbol{k})=\sum_{\substack{\boldsymbol{p}, \boldsymbol{q} \\
k=\boldsymbol{q}+q}} \sum_{i, j} \Gamma_{A_{j} A_{i}}(\boldsymbol{k}, \boldsymbol{p}, \boldsymbol{q})\left[\hat{a}_{i}(\boldsymbol{p}) \hat{a}_{j}(\boldsymbol{q})+\hat{a}_{j}(\boldsymbol{p}) \hat{a}_{i}(\boldsymbol{q})\right],
\end{gathered}
$$

where the explicit expression of the coefficients $\Gamma_{i, j}(\boldsymbol{k}, \boldsymbol{p}, \boldsymbol{q})$ can be found from the nonlinear terms in (2.5). The interaction terms including barotropic modes have to be treated separately, since we decompose the flow field into $q, w$ and $b$ for these modes. 
Multiplying (2.15) by $\hat{q}^{*}(\boldsymbol{k}) / 2 k^{2}$, we obtain the geostrophic energy budget

$$
\frac{\partial E_{G}(\boldsymbol{k})}{\partial t}=T_{G G G}(\boldsymbol{k})+T_{G G A}(\boldsymbol{k})+T_{G A A}(\boldsymbol{k}),
$$

where $T_{G G G}, T_{G G A}$ and $T_{G A A}$ represent the transfers into geostrophic energy due to nonlinear interactions involving two geostrophic, one geostrophic and one ageostrophic and two ageostrophic modes, respectively. In a similar way we arrive at an expression for $E_{A}$,

$$
\frac{\partial E_{A}(\boldsymbol{k})}{\partial t}=T_{A G G}(\boldsymbol{k})+T_{A G A}(\boldsymbol{k})+T_{A A A}(\boldsymbol{k}) .
$$

The transfer functions satisfy the following conservation relations

$$
\begin{aligned}
\sum_{k} T_{G G G}(\boldsymbol{k}) & =0, \\
\sum_{\boldsymbol{k}} T_{G G A}(\boldsymbol{k})+T_{A G G}(\boldsymbol{k}) & =0, \\
\sum_{\boldsymbol{k}} T_{A G A}(\boldsymbol{k})+T_{G A A}(\boldsymbol{k}) & =0, \\
\sum_{\boldsymbol{k}} T_{A A A}(\boldsymbol{k}) & =0, \\
\sum_{\boldsymbol{k}} k^{2} T_{G G G}(\boldsymbol{k}) & =0,
\end{aligned}
$$

where the last relation is an expression of the fact that potential vorticity is conserved by interaction involving only geostrophic modes. From the energy transfer functions, it is also straightforward to define the enstrophy transfer functions. Since the enstrophy spectrum is given by

$$
Q(\boldsymbol{k})=\frac{\hat{q}(\boldsymbol{k}) \hat{q}^{*}(\boldsymbol{k})}{2},
$$

the enstrophy transfer functions can easily be found from (2.17) as

$$
T_{G G G}^{\eta}(\boldsymbol{k})=k^{2} T_{G G G}(\boldsymbol{k}), \quad T_{G G A}^{\eta}(\boldsymbol{k})=k^{2} T_{G G A}(\boldsymbol{k}), \quad T_{G A A}^{\eta}(\boldsymbol{k})=k^{2} T_{G A A}(\boldsymbol{k}) .
$$

The transfer functions give information about the amount of energy/enstrophy flowing into or out of a certain mode. Nevertheless, they do not preserve the information about where such energy comes from and which wavenumbers are involved in the exchange. In order to shed some light on the dynamics physical process, such kind of information is however crucial. Therefore we will also consider the triad interaction terms as functions of $k=|\boldsymbol{k}|, p=|\boldsymbol{p}|$ and $q=|\boldsymbol{q}|$. For example, we denote by $T_{G G G}(k, p, q)$ the transfer function which is calculated by averaging the relevant triad interactions over $k, p$ and $q$. Note that according to the definitions, such transfer functions are symmetric with respect to $p$ and $q$.

The energy/enstrophy flux

$$
\Pi(k)=-\sum_{|k|=0}^{|k|=k} T(\boldsymbol{k}) \quad \text { and } \quad \Pi^{\eta}(k)=-\sum_{|k|=0}^{|k|=k} T^{\eta}(\boldsymbol{k})
$$


are often used in place of their generic transfer function $T(\boldsymbol{k})$ and $T^{\eta}(\boldsymbol{k})$. It is therefore quite natural to classify the fluxes in a similar manner as done for the transfer terms. In particular, a special attention will be paid to the energy and enstrophy fluxes due to the geostrophic interactions only,

$$
\Pi_{G}(k)=-\sum_{|k|=0}^{|k|=k} T_{G G G}(\boldsymbol{k}) \quad \text { and } \quad \Pi_{G}^{\eta}(k)=-\sum_{|\boldsymbol{k}|=0}^{|\boldsymbol{k}|=k} T_{G G G}^{\eta}(\boldsymbol{k}) .
$$

A large amount of literature has recently focused on the relative importance of horizontally divergent and rotational motions, both in the context of stratified turbulence (Lelong \& Riley 1991; Lindborg \& Brethouwer 2007) and mesoscale dynamics (Lindborg 2007; Hamilton et al. 2008; Waite \& Snyder 2009; Molemaker et al. 2010). Even though it is not clear how meaningful the rotational/divergent decomposition is for geophysical dynamics, its importance indeed relies on the fact that it can be applied to observational data (Lindborg 2007). Also, such a decomposition is often used to present results from global circulating models (Hamilton et al. 2008). Following Lelong \& Riley (1991), we may rewrite the velocity as

$$
\boldsymbol{u}=\boldsymbol{e}_{z} \times \nabla_{h} \psi+\left\{\nabla_{h} \phi+w \boldsymbol{e}_{z}\right\},
$$

where the first term is horizontally non-divergent whereas the second carries all of the horizontal divergence of the velocity field. The rotational part of the velocity field has two contributions, one which is associated with $q$ and another which is associated with $a_{1}$ and $a_{2}$, whereas the divergent part of the velocity field is only associated with $a_{1}$ and $a_{2}$.

\section{Simulations}

\subsection{Numerical methodology}

The system (2.5) is discretized in a triply-periodic (the adjective triply is referred to periodic not to the domain) isotropic domain, allowing for Fourier representation of the variables in all three spatial directions. Observe that the box is cubic in a space where the vertical coordinate is stretched by a factor of $N / f$. A pseudospectral method is used, providing an exponential convergence of the numerical solution. Nonlinear terms in (2.5) are advanced in time using a low-storage fourthorder Runge-Kutta scheme. Linear terms are instead separately solved using an exact implicit procedure (Canuto et al. 1988). In order to prevent aliasing errors, the 2/3dealiasing rule was applied to nonlinear terms (2.5).

Velocities and buoyancy are recovered from $q, a_{1}$ and $a_{2}$, using the inversion relations (2.6). However, for $k_{z}=0$, the inversion relations (2.6) becomes singular and, as shown by (2.2b) and (2.2c), $a_{1}$ and $a_{2}$ are not independent, i.e. $a_{1, y}=a_{2, x}$. For the mode $k_{z}=0$ we instead solve for $q, w$ and $b$, using the $(2.5 a),(2.1 b)$ and (2.1c), respectively. The horizontal velocity components can then be recovered from the potential vorticity inversion

$$
\nabla^{2} \boldsymbol{u}_{h}=-\nabla \times q \boldsymbol{e}_{z},
$$

whereas $a_{1}$ and $a_{2}$ are calculated from their definitions, (2.2b) and (2.2c), respectively.

A random forcing is introduced in the $q$ equation only, i.e. no ageostrophic motions are directly forced. The forcing scheme is the same as used by Vallgren \& Lindborg (2011). The scheme is white noise in time, so that no particular time scale is forced. 


$\begin{array}{lcccccccc}\text { Run } & R o & F r & v_{L} & v_{S} \times 10^{18} & \varepsilon_{L} / P & \varepsilon_{S} / P & \Delta t^{T S} & T^{T S} \\ \text { PE2 } & 0.2 & 0 & 0.012 & 6.2 & 0.30 & 0.36 & - & - \\ \text { PE1 } & 0.1 & 0 & 0.012 & 4.0 & 0.43 & 0.14 & - & - \\ \text { PE05 } & 0.05 & 0 & 0.012 & 4.0 & 0.46 & 0.05 & - & - \\ \text { PE025 } & 0.025 & 0 & 0.012 & 4.0 & 0.25 & 7.5 \times 10^{-3} & - & - \\ \text { PE0 } & 0 & 0 & 0.012 & 4.0 & 0.43 & 5.4 \times 10^{-4} & - & - \\ \text { aBQ2 } & 0.2 & 0.01 & 0.012 & 6.2 & 0.43 & 0.27 & - & - \\ \text { aBQ1 } & 0.1 & 0.01 & 0.012 & 4.0 & 0.76 & 0.08 & - & - \\ \text { aBQ05 } & 0.05 & 0.01 & 0.012 & 4.0 & 0.70 & 5.9 \times 10^{-3} & - & - \\ \text { bBQ001 } & 0.1 & 0.001 & 0.012 & 6.2 & 0.72 & 0.086 & 1.4 \times 10^{-3} & 3.20 \\ \text { bBQ01 } & 0.1 & 0.01 & 0.012 & 4.0 & 0.76 & 0.08 & 1.9 \times 10^{-2} & 18.3 \\ \text { bBQ1 } & 0.1 & 0.1 & 0.012 & 4.0 & 0.86 & 0.064 & 1.8 \times 10^{-2} & 20.3 \\ & & & & & & & & \\ \text { ST } & - & - & 0.012 & 20 & 0.06 & 0.90 & - & -\end{array}$

TABLE 1. Summary of the simulations. The physical parameters have been calculated after a steady direct cascade was established. Large- and small-scale dissipation have been made dimensionless with respect to the energy injection rate $P$. Note that for most of the runs, energy was still growing due to the inverse cascade.

The forcing is perfectly decorrelated to the velocity field, allowing us to exactly control the enstrophy and the energy injection rates into the system. The enstrophy injection rate, $\eta$, is set to unity in all of the simulations, which means that we can regard time as non-dimensionalized using $\eta^{1 / 3}$. The forcing is isotropic in the vertically-stretched (vertically is referred to stretched) space and is applied to large scales only, corresponding to the wavenumber band $k \in[3,5]$. The forcing has a Gaussian distribution over this range. The simulations are initialized with random flow fields and they are run long enough for a steady energy cascade to develop.

To allow for very high-resolution simulations, the numerical code was parallelized with the use of the message passing instratified turbulence terface (MPI) and run on up to 4096 processors, resulting in a linear scalability. Inviscid energy conservation tests, in agreement with (2.7), have been carried out without applying any forcing and providing an increasing conservation of energy up to machine precision as time step was progressively reduced. In the limit of zero Ro, results in agreement with QG numerical simulations (Vallgren \& Lindborg 2011) were obtained.

\subsection{Choice of the numerical parameters}

The numerical and physical parameters used in the simulations are listed in table 1. The box is chosen as $\left(L_{x} \times L_{y} \times L_{z}\right)=(2 \pi \times 2 \pi \times 2 \pi)$, using 1024 modes in each direction. The physical parameters in table 1 were extracted from the simulations after a steady direct energy cascade was obtained. The values of $R o$ and $F r$ were chosen to span a realistic range representative for atmospheric applications. Boer \& Shepherd (1983) estimated the enstrophy flux from global FGGE data, giving a value of the order of $10^{-15} \mathrm{~s}^{-3}$. Cho \& Lindborg (2001) made a similar estimate using the third-order structure functions measured in the lower stratosphere. Such a value gives a Rossby number, $R o=\eta^{1 / 3} / f$, of the order of 0.1 for midlatitude dynamics. A realistic value of $N$ is of the order of $10^{-2} \mathrm{~s}^{-2}$ (Vallis 2006), corresponding to a ratio, $f / N$, of $\sim 0.01$. With the aim of reproducing dynamics representative of the atmosphere, we have carried out simulations with $f / N=F r / \operatorname{Ro} \in[0,1]$. As shown 
(a)

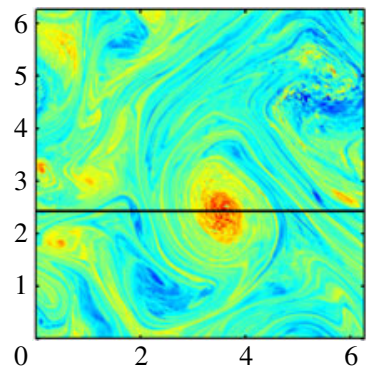

(b)

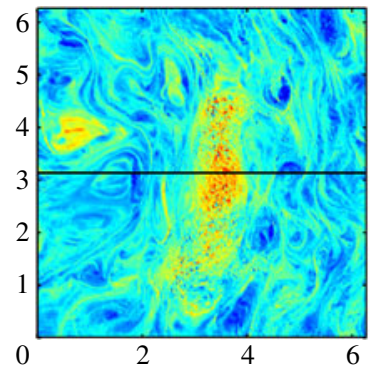

(c)



FIGURE 1. Potential vorticity $q(a, b)$ and the second ageostrophic component $a_{2}(c)$ cuts for the aBQ1 run. $(a, c)$ Horizontal cuts at $z=3.14 ;(b)$ vertical cut at $y=2.4$, corresponding to the location of the large-scale vortex. Dark helping lines show the position of the vertical and horizontal cut planes.

by Vallgren et al. (2011), in the limit of zero Fr, an increasing amount of energy cascades towards small scales as $R o$ is increased. Therefore, in order to keep the dissipation range well-resolved, the small-scale viscosity has to be somewhat increased with increasing $R o$.

We divide the simulations into four sets called PE, aBQ, bBQ and ST. Each run is named accordingly, followed by a number $x$. In the PE simulations, $F r$ is set to zero and $x$ refers to the Rossby number which is varied between 0 and 0.2 . In the aBQ runs, $F r$ is set to 0.01 and the Rossby number, indicated by the suffix $x$, is varied in the same way as in the PE set. In the bBQ, Ro is instead kept fixed to 0.1 , whereas the Froude number, indicated by $x$, is varied from 0.001 to 0.1 . Finally, a non-rotating simulation of the PEs has also been run and named ST in order to investigate the vertical spectrum, as discussed in $\S 5$. For the ST runs, the grid spacing is stretched by a factor $F r^{-1}$ in accordance to the scaling proposed by Billant \& Chomaz (2001) for stratified flows. The computational grid is isotropic in the space where the vertical coordinate has been stretched. In order to investigate the role of gravity waves and inertial waves, time series of individual Fourier modes are collected for the bBQ set after that a forward cascade is established. The sampling time step $\Delta t^{T S}$ and the covered time interval $T^{T S}$ are given in table 1.

\section{Flow fields}

We start by presenting some snapshots from the simulations in order to give a feeling of the physical process that we investigate. In figure 1(a), a horizontal cut of the $q$ flow field is shown for the aBQ1 run, i.e. $R o=0.1$ and $F r=0.01$. The overall dynamics at large-scales resemble the QG dynamics (see for instance figure 9 of Vallgren \& Lindborg (2010)) with a large-scale vortex surrounded by small-scale filaments. Figure 1(b) displays a vertical cut of the large-scale vortex. Interestingly, this structure shows a large degree of coherence in the vertical direction, displaying similarities with the large-scales vortices found by Vallgren \& Lindborg (2010) and the related barotropization of the flow. Nevertheless, some differences can also be observed. Unlike the QG simulation results, the core of the vortex is dominated by small-scale structures which can be observed both in the horizontal and in the vertical cuts. Moreover, patches of small-scale turbulence can also be observed all over the flow. A particularly intense small-scale chaotic region is found in the top right corner of the horizontal $q$ cut in figure 1(a). As shown by the horizontal cut of $a_{2}$ in 
(a)

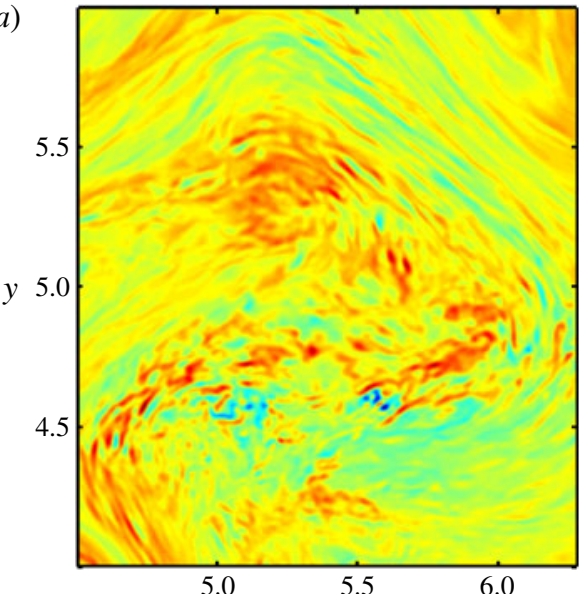

(c)

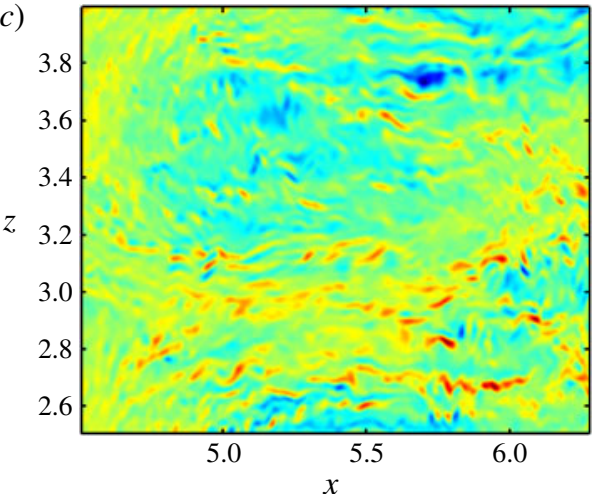

(b)

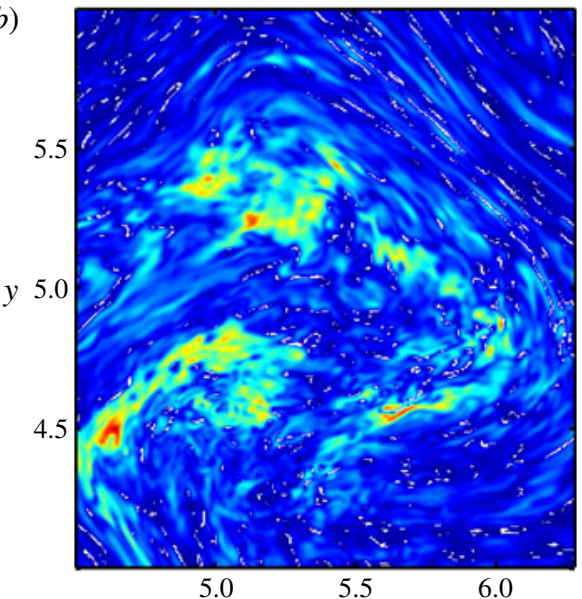

$(d)$

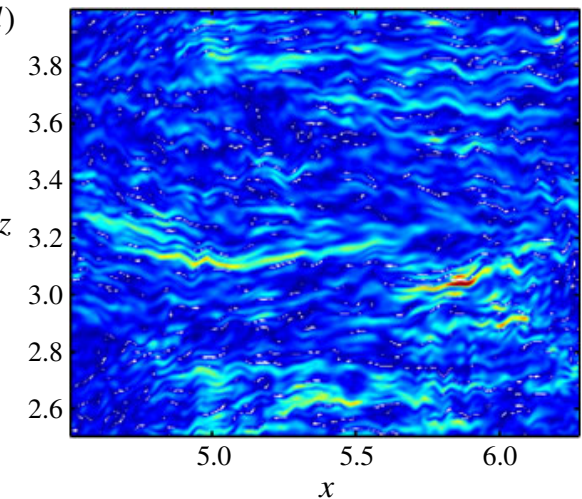

FIgURE 2. Close-up of the turbulent patch in the right top corner of figure $1(a)$ (aBQ1 r). $(a, c)$ Potential vorticity. $(b, d)$ Local Froude number $F r_{L} .(a, b)$ Horizontal cuts. $(c, d)$ Vertical cuts. The colourmap of the local Froude number ranges from 0 (blue) to 1.8 (red).

figure $1(c)$, in such a region the ageostrophic motions become more intense than in other regions, increasing of about one order of magnitude.

Molemaker et al. (2010) reported snapshots of their non-hydrostatic non-geostrophic forced Eady flow, where characteristic structures of QG dynamics as filaments and small-scale three-dimensional turbulence were both observed. They argued that a forward energy cascade is needed for the system to reach a balanced state when a constant energy input is introduced into the flow. They showed that dissipative turbulent patches arise as the result of instabilities developing along potential vorticity fronts. The horizontal and vertical structures of the turbulent patches in figures $1(a)$ and $1(b)$ are illustrated in the close-up shown in figure 2, where both the $q$-field and the local vertical Froude number, defined as

$$
F r_{L}=\frac{\sqrt{\omega_{x}^{2}+\omega_{y}^{2}}}{2 N},
$$

are shown in an horizontal (parallel to the $x$-direction) and vertical plane. In (4.1), $\omega_{x}$ and $\omega_{y}$ represent the dimensional horizontal vorticities. In the horizontal plane, $q$ and 
(a)

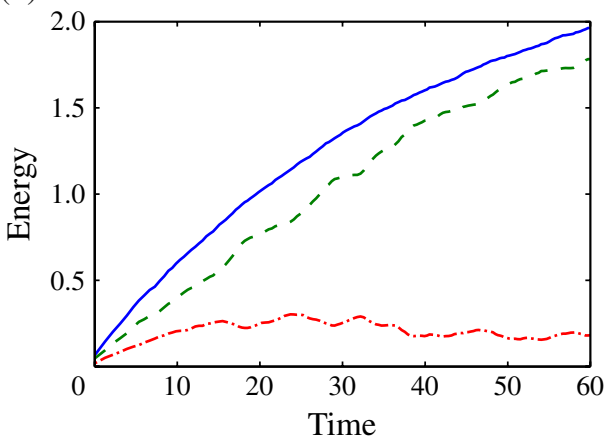

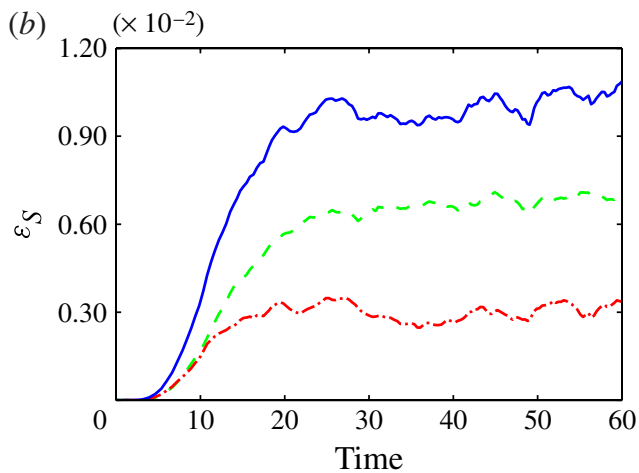

FIgURE 3. (Colour online) (a) Time evolution for the energy and (b) the small-scale energy dissipation for the aPE1 run: __, total;,--- , kinetic;.,-- , potential. Time is made dimensionless with the enstrophy injection rate $\eta$. Energy is made dimensionless with $\eta^{2 / 3} / k_{0}^{2}$.

$F r_{L}$ show small-scale structures which follow very similar patterns. More importantly, $\mathrm{Fr}_{L}$ attains values of the order of unity, which is required for $\mathrm{KH}$ instabilities to develop (Billant \& Chomaz 2001). Very similar observations can also be made in the vertical plane. It is also worth noting how confined the structures in the vertical direction are as compared with the horizontal direction (figure $2 c, d$ ).

\section{Energy spectra and spectral fluxes}

\subsection{Horizontal spectra}

Simulations were run long enough to allow for the formation of a steady downscale energy cascade with a nearly constant small-scale dissipation. Figure 3(a) shows the time evolution of both kinetic and potential energy in the run aPE1. Qualitatively, the same picture was obtained in all of the other runs. To start with, the kinetic energy grows twice as fast as the potential energy, as an effect of the Charney isotropic forcing (Vallgren \& Lindborg 2010). However, whereas potential energy saturates quickly at very early stages, kinetic energy continues to increase and levels off only at a later stage. In the quasi-steady state, there is considerably more kinetic energy than potential energy. Similar results were also obtained by Vallgren \& Lindborg (2011) in QG simulations. However, kinetic energy tends to level off at earlier times as Ro is increased. In spite of the fact that the kinetic energy is still growing, small-scale dissipation attains nearly constant values rather soon (figure $3 b$ ), at times comparable to the time required by the potential energy to saturate. For the $R o$ considered in figure 3, the kinetic energy dissipation is observed to be larger than potential energy dissipation.

We begin by examining the horizontal two-dimensional energy spectra, shown in figure 4 , both for the PE $(a)$ and aBQ $(b)$ sets. The spectra were calculated by averaging over circles with constant $k_{h}=\sqrt{k_{x}^{2}+k_{y}^{2}}$. The curves in figure 4(a) compare well with the results of Vallgren et al. (2011), who studied the one-dimensional horizontal spectra. The spectra at low $R o$ scale as $k_{h}^{-3}$ for a large span of wavenumbers. As $R o$ is increased, departures from the $k_{h}^{-3}$ dependence are observed at the large wavenumber end and slopes close to $-5 / 3$ at small scales are attained (Bartello 2010). Consistent with the results of Vallgren et al. (2011), spectra for both PE and BQ sets 

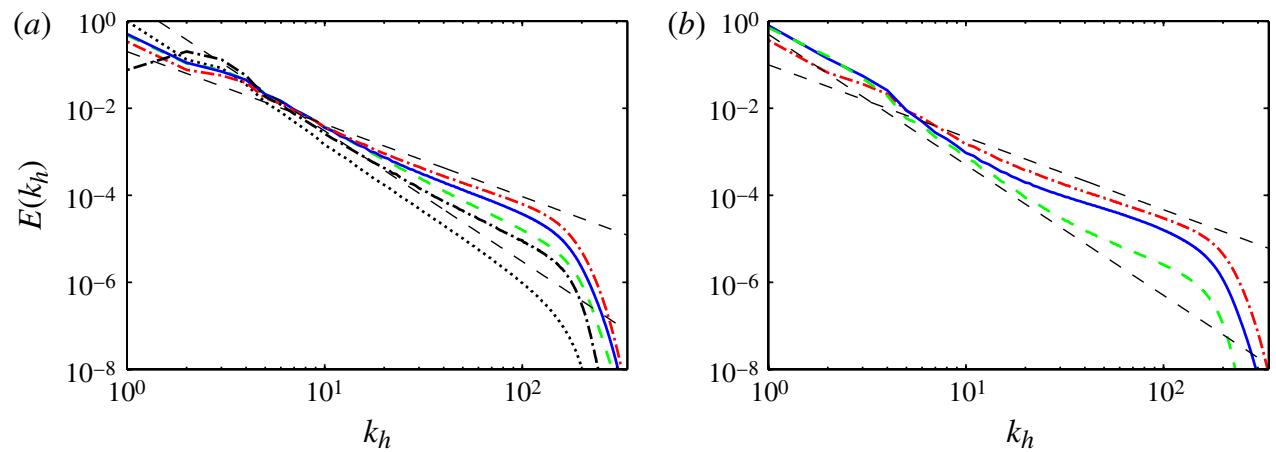

FIgure 4. (Colour online) Comparison of the two-dimensional horizontal total energy spectra for (a) PE and (b) aBQ runs for several Ro numbers:.,$- R_{o}=0.2 ;-, R o=0.1$; ,$--- R o=0.05 ;-.-, R o=0.025 ; \cdots \cdots, R o=0.0$ (only PE runs). The --- are helping lines with $k_{h}^{-5 / 3}$ and $k_{h}^{-3}$.
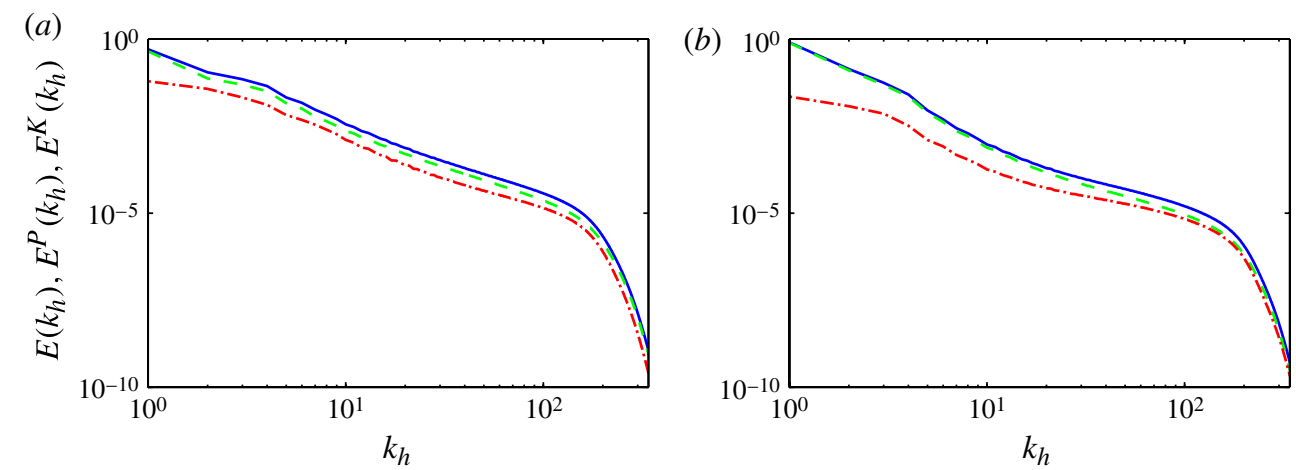

FIgURE 5. (Colour online) Kinetic and potential energy spectra for the $(a)$ PE and $(b)$ aBQ runs with $R o=0.1$ : —

smoothly tend to the QG limit as $R o$ is reduced, with the transition to $k^{-5 / 3}$ being pushed towards larger wavenumbers. This is a direct consequence of the increasing amount of energy which cascades downscale with increasing Ro. As we will see (in figure $10 b$ ), in the PE simulations the energy cascade is somewhat stronger for a given $R o$ than in the $\mathrm{BQ}$ simulations. For a given $R o$ the transition wavenumber is therefore smaller in the PE simulations as compared with the BQ simulations, even though a qualitatively similar behaviour can be observed for PE and BQ simulations.

As shown by figure 5, at large scales kinetic energy dominates over the potential energy and it accounts almost completely for the total energy content. Similar results were also found in numerical simulations of the QG equation (Vallgren \& Lindborg 2011). However, it should also be noted that the gap between kinetic and potential energy tends to increase in the BQ runs as compared with the PE runs and equipartition occurs only at small scales. 

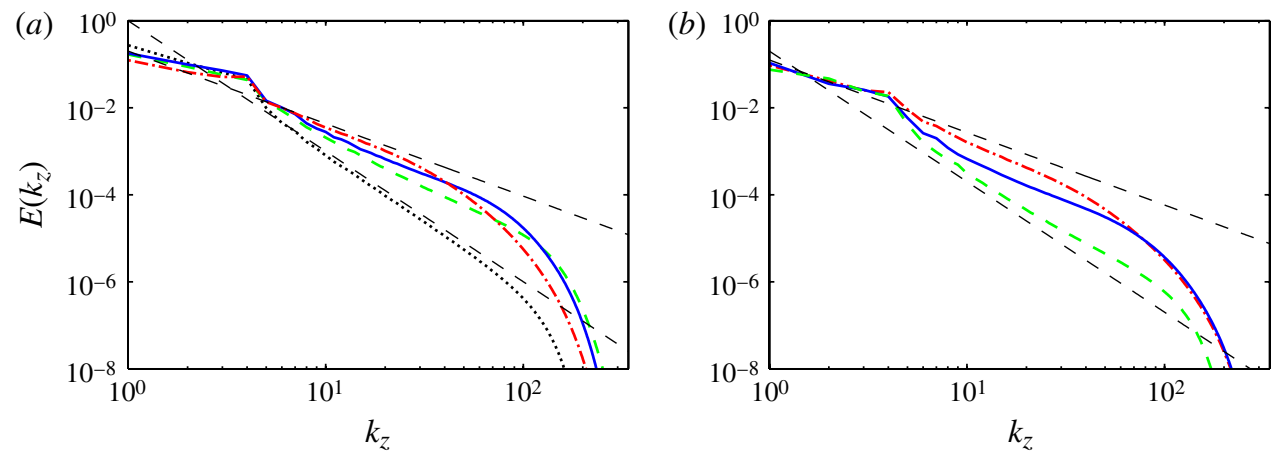

FIGURE 6. (Colour online) Comparison of the one-dimensional vertical total energy spectra for $(a)$ PE and (b) aBQ runs for several Ro numbers:.,- Ro $=0.2 ;-, R o=0.1 ;----$, $R o=0.05 ; \cdots \cdots, R o=0.0$ (only PE runs). The --- are helping lines with $k_{h}^{-5 / 3}$ and $k_{h}^{-3}$.

\subsection{Vertical spectra}

We now turn to the vertical spectra, shown in figure 6. In a similar way as the horizontal spectra, the vertical spectra display many similarities between PE and BQ runs as $R o$ is increased. As reported in QG literature, also in our simulations the inverse cascade of energy leads to an accumulation of energy in the barotropic mode, i.e. $k_{z}=0$. The amount of energy which is contained in this mode is quite significant, especially for BQ runs where it varies from 60 to $80 \%$ of the total, increasing as $R o$ reduces. In $\mathrm{QG}$ dynamics, the vertical spectrum has a similar form, $\sim \eta^{2 / 3} k_{z}^{-3}$, as the horizontal spectrum. In the current simulations, the slopes are found to increase with $R o$ from -3 to $-5 / 3$. We find it somewhat surprising that the vertical spectra also show such a transition. In contrast to this result, the vertical spectra of strongly stratified turbulence which is not affected by system rotation is expected to scale as $k_{z}^{-3}$ (Billant \& Chomaz 2001). In order to investigate whether this is the case, we carried out an additional simulation of stratified turbulence (the ST run). In order to recover the purely stratified regime the linear terms pertaining to the Coriolis forces in (2.1) are set to zero. Moreover, setting $R o$ to unity in (2.3) allows us to retrieve the scaling for strongly stratified flows suggested by Billant \& Chomaz (2001). Hydrostasy was assumed in the vertical by imposing $F r$ equal to zero. In figure 7(a) the vertical spectrum for this simulation is shown together with the vertical spectrum pertaining to the PE1 run. In agreement with previous studies in the field of stratified turbulence, a spectrum of the form

$$
E\left(\tilde{k}_{z}\right)=C N^{2} \tilde{k}_{z}^{-3}
$$

is recovered, with $C \approx 1$. Here $\tilde{k}_{z}$ is the dimensional vertical wavenumber. This result suggests that the shallowing of the vertical spectrum is an effect of the system rotation.

Vallgren \& Lindborg (2011) tested the validity of Charney isotropy (Charney 1971) in high-resolution numerical simulations of QG turbulence. They found that the ratio between the one-dimensional vertical spectrum and the corresponding horizontal spectrum

$$
R(k)=\frac{E_{z}(k)}{E_{h}(k)},
$$




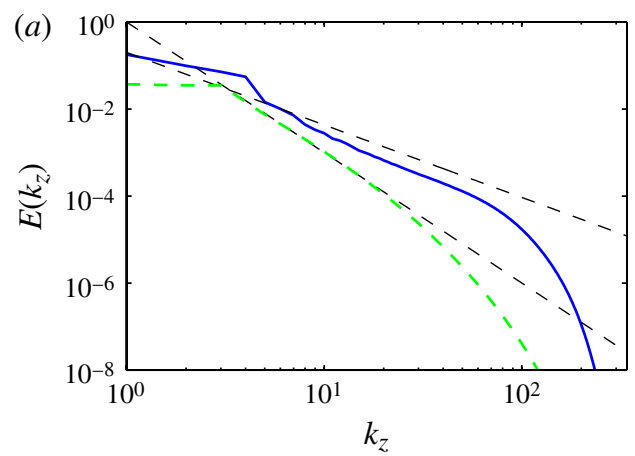

(b)

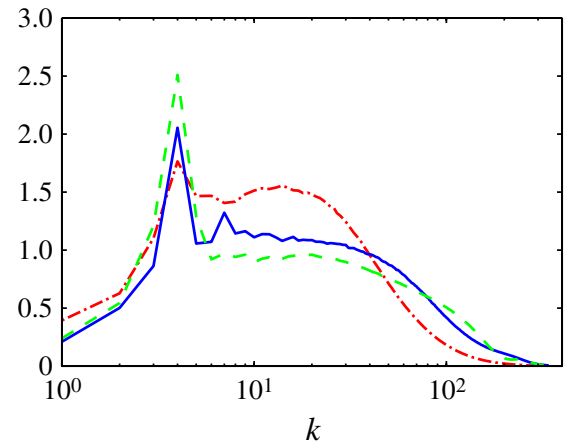

FIgure 7. (Colour online) (a) Comparison of vertical spectra for PEs with rotation $R o=0.1$ PE1 (_- ) and without rotation ST (--- ). (b) Measure of the Charney isotropy according to (5.2) for several $R o$ for the aBQ runs: ${ }_{-} .$, , $R o=0.2 ; \_, R o=0.1 ;{ }_{----}, R o=0.05$.

is approximately equal to unity, except at the forcing and dissipating scales. In (5.2), $E_{h}(k)$ is the one-dimensional horizontal spectrum and $E_{z}(k)$ is the one-dimensional vertical spectrum, where $k$ in the vertical spectrum is the vertical wavenumber stretched by a factor of $f / N$. As $R o$ is increased, Charney isotropy is expected to apply to a smaller degree. In figure $7(b), R(k)$ is plotted for several $R o$. The peak at $k=4$ is an artefact of the forcing. For larger wavenumber, a plateau is observed in all cases. Comparing figure $7(a)$ and $(b)$, we see that the plateau, in fact, extends into the range where the spectrum scales as $k^{-5 / 3}$. This is a quite unexpected results. However, the width of the plateau seems to decrease with increasing $R o$, whereas its magnitude increases with $R o$.

\subsection{Rotational and divergent contributions}

Since the results of the PE and BQ simulations are qualitatively very similar as $R o$ is changed, henceforth in this subsection we focus only on PE runs. Results from BQ $x$ runs do not differ qualitatively, and analogous conclusions would therefore apply. A question yet to be answered regarding the atmospheric kinetic energy spectrum and its transition from $k_{h}^{-3}$ to $k_{h}^{-5 / 3}$ at scales of $\sim 500 \mathrm{~km}$ concerns the importance of rotational and divergent motions. In the QG limit, the divergent part vanishes due to the incompressibility condition (2.1d). At large scales, where QG is a good approximation, the rotational part should therefore be dominant. Lindborg (2007) calculated the rotational and divergent energy spectra from measurements in the upper troposphere and lower stratosphere and found that rotational modes are totally dominant at synoptic scales but the contributions from the two types of modes are of the same order of magnitude at mesoscales. Lindborg \& Brethouwer (2007) found that energy is equipartitioned between rotational and divergent modes in the turbulent cascade of strongly stratified flows. In figure $8(a)$, the kinetic energy spectrum is shown together with the rotational and divergent contributions. At large scales, the rotational part dominates, whereas the divergent part is several orders of magnitude smaller. The rotational spectrum scales as $k_{h}^{-3}$ in this range. On the other hand, the divergent energy spectrum is rather flat, a little shallower than $k_{h}^{-5 / 3}$. Owing to the different slopes, rotational and divergent energy spectra are doomed to meet at a transition wavenumber $k_{t r}$. As this scale is approached, the rotational spectrum shallows whereas the divergent spectrum steepens slightly and both tend to $\sim k_{h}^{-5 / 3}$. 

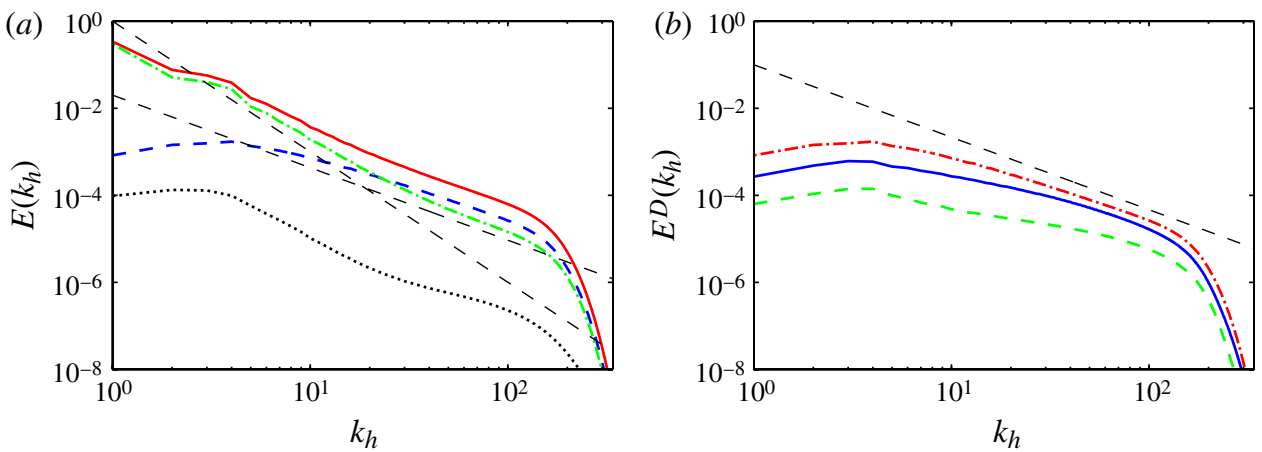

FIGURE 8. (Colour online) (a) Divergent (---_) and rotational (-.-) part of the kinetic energy (—) for the PE2 run. The QG divergent energy, given by (5.3), is shown as well for reference $(\cdots \cdots)$. The --- are helping lines with $k_{h}^{-5 / 3}$ and $k_{h}^{-3} .(b)$ Divergent energy for several $R o$ for the PE runs: $\_.-, R o=0.2 ; \_, R o=0.1 ;-\ldots, R o=0.05$. The ${ }_{-}-{ }_{-}$are helping lines with $k_{h}^{-5 / 3}$.

For higher wavenumbers, the energy content in rotational and divergent modes is of the same order of magnitude, consistent with the results on stratified turbulence of Lindborg (2007) and Lindborg \& Brethouwer (2007). The ratio between the divergent and the rotational energy $E_{D} / E_{R}$ is small at large scales and attains a value of the order of two after the transition of the spectrum.

Setting $R o=0$ and $F r=0$ in (2.5b) and (2.5c), one may derive the so-called $\Omega$-equation for the vertical velocity (e.g. Gill 1982),

$$
\begin{aligned}
\nabla^{2} w= & -\frac{\partial^{2}}{\partial y \partial z}\left(\frac{\partial u^{2}}{\partial x}+\frac{\partial u v}{\partial y}\right)+\frac{\partial^{2}}{\partial x \partial z}\left(\frac{\partial u v}{\partial x}+\frac{\partial v^{2}}{\partial y}\right) \\
& -\left(\frac{\partial^{2}}{\partial x^{2}}+\frac{\partial^{2}}{\partial y^{2}}\right)\left(\frac{\partial u b}{\partial x}+\frac{\partial v b}{\partial y}\right) .
\end{aligned}
$$

An interesting question is whether the divergent spectrum which is obtained by solving the $\Omega$-equation is comparable to the total divergent spectrum. In figure $8(a)$ we investigate this. The divergent spectrum which is obtained from (5.3) is several orders of magnitude smaller than the total divergent spectrum. This clearly shows that the transition from $\sim k_{h}^{-3}$ to $\sim k_{h}^{-5 / 3}$ cannot be explained within a higher-order QG model. Whether this is also the case in the atmosphere is, to the best of the authors' knowledge, not clear. Unfortunately, the data used by Lindborg (2007) did not allow the geostrophic divergent part to be computed. Notwithstanding, the fact that at these scales rotational and divergent parts are of the same order of magnitude suggests indeed that QG dynamics is not the only dominant one.

The Rossby number dependence of the divergent energy spectrum is investigated in figure $8(b)$. As is clear from the plot, an increase of Ro leads to a larger amount of divergent energy. It can be argued that this energy is directly linked to the forward energy cascade whose strength increases with $R o$. Note that for small Ro the slopes are somewhat shallower than $-5 / 3$ at small wavenumbers but tend to steepen with increasing wavenumber.

It can be of interest to compare the divergent/rotational decomposition with the geostrophic/ageostrophic decomposition. As pointed out in $\S 2$, the two decompositions 

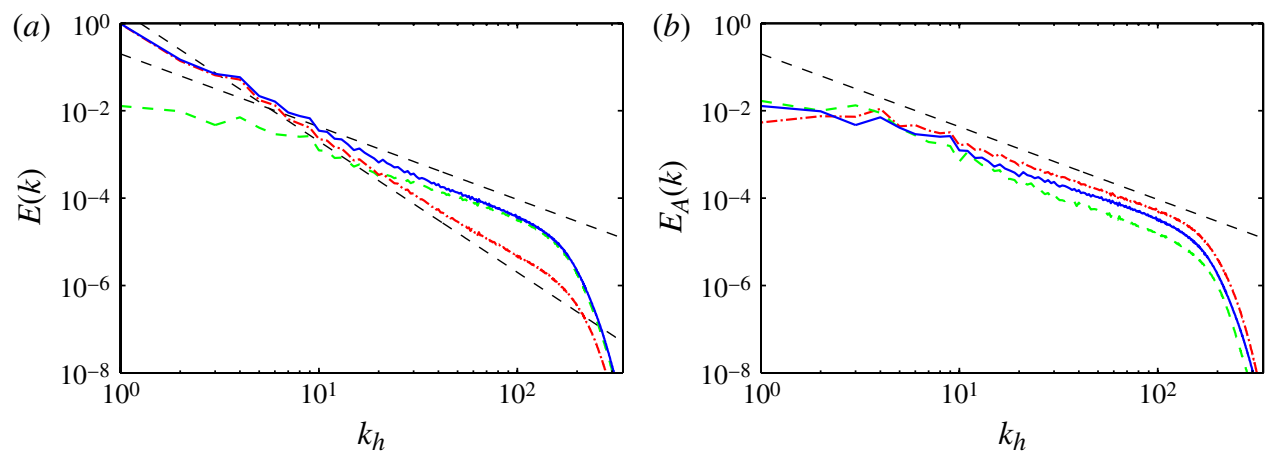

Figure 9. (Colour online) (a) Geostrophic (-- - ) and ageostrophic (-.-) part of the total energy spectrum (_- ) for the PE2 run. The - - - are helping lines with $k_{h}^{-5 / 3}$ and $k_{h}^{-3} .(b)$ Ageostrophic energy for several $R o$ for the PE runs:.,$-- R o=0.2 ;-, R o=0.1 ;-{ }_{---}$, $R o=0.05$. The --- are helping lines with $k_{h}^{-5 / 3}$.

are not equivalent. If conditions are reasonably close to QG dynamics, the contribution of ageostrophic modes to the rotational part is of the order Ro smaller. On the other hand, larger differences should be expected at scales at which the geostrophic scaling (2.3) is not valid. In figure $9(a)$ the total energy spectrum pertaining to the PE2 run is decomposed into its geostrophic and ageostrophic parts. Consistent with the results of Bartello (1995, 2010), at large scales the dominant contribution comes from the geostrophic component whereas at small scales the ageostrophic energy is the largest. The ageostrophic spectrum attains a constant scaling (close to $-5 / 3$ ) over the whole range of scales. Interestingly, this holds to a slightly lesser extent also for the geostrophic energy spectrum which shows a $k^{-3}$ dependence over a wide range. Indeed, the $k^{-3}$ scaling is remarkably attained at wavenumbers larger than the transition wavenumber. In figure $9(b)$ the ageostrophic spectrum is shown for the PE set. By comparing figure $8(b)$ with figure $9(b)$ two differences can mainly be observed. First, the large-scale divergent spectrum shows a somewhat shallower behaviour. Second, the Ro dependence of the magnitude of the spectrum is stronger for divergent energy as compared with ageostrophic energy.

\subsection{Energy and enstrophy fluxes}

Vallgren et al. (2011) showed that within the PE framework, the amount of energy cascading towards small scales is a function of $R o$. In particular, they found that the small-scale dissipation scaled as $\varepsilon_{S} \sim R o^{3 / 2} P$, where $P$ is the energy injection. Here, we extend their analysis and investigate the influence of a non-zero $F r$. The total energy flux (2.22) is plotted in figure $10(a)$ for the $\mathrm{aBQ} x$ runs. In agreement with the observations of Vallgren et al. (2011), the amount of energy cascading towards small-scales increases with $R o$. Notwithstanding, the quantitative magnitude and the $R o$ dependence are different. In figure 11 the small-scale dissipation $\varepsilon_{S}$ is shown for $\mathrm{PE}$ and aBQ sets, spanning values of $R o$ between 0.025 and 0.2 . The QG energy flux is found to be several orders of magnitude smaller (Vallgren et al. 2011). Despite the rather small values of $R o$ considered in the study, a consistent fraction of energy is found to cascade towards small scales. At $R o=0.1, \varepsilon$ is almost $10 \%$ of the total injection of energy $P$ (see table 1 ). As can be clearly seen, the departure from the hydrostatic approximation lead to somewhat smaller energy fluxes towards small 

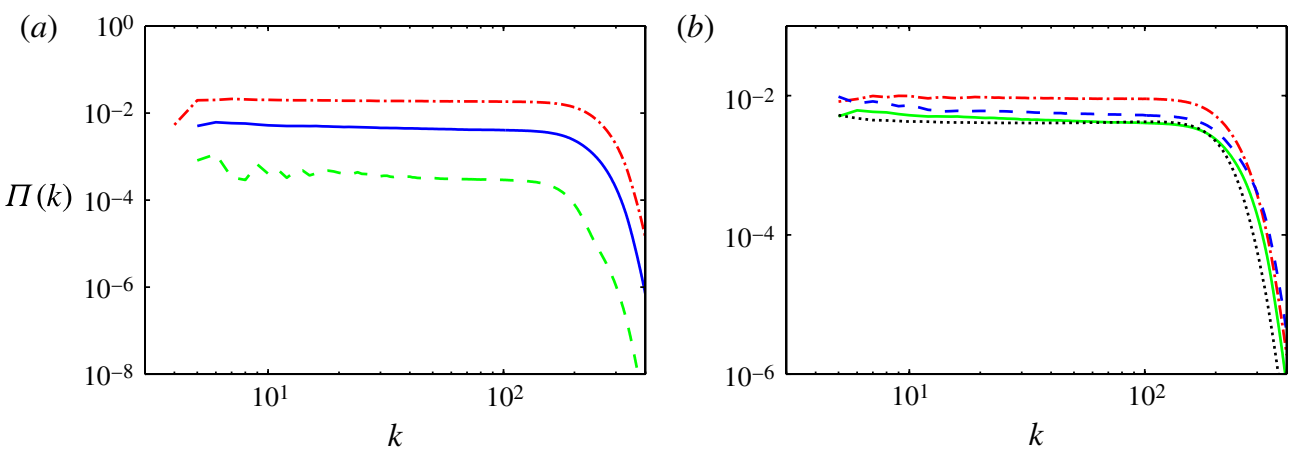

FIGURE 10. (Colour online) Total energy flux function of the wavenumber $k$ for $(a)$ aBQ and (b) bBQ runs: $(a)-.-, R o=0.2 ; \_, R o=0.1 ;---, R o=0.05 ;(b)-.-, F r=0 ;----$, Fr $=0.001 ;-, F r=0.01 ; \cdots \cdots, F r=0.1$.

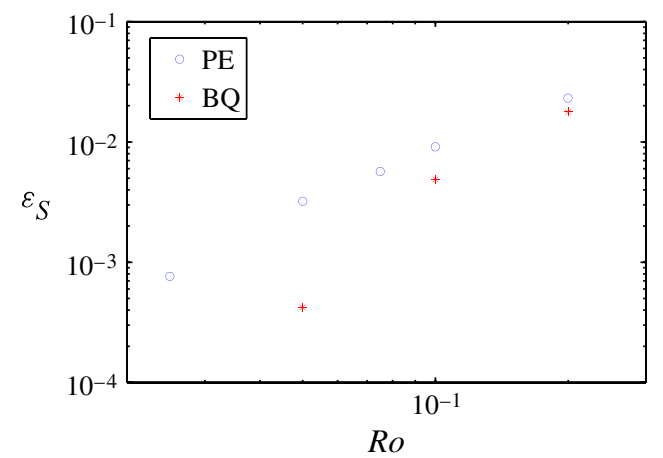

FIGURE 11. (Colour online) Small-scale dissipation function of the Ro, both for PE $(\bigcirc)$ and aBQ (*) runs. For the aBQ runs the $F r$ number has been kept constant and equal to 0.01 .

scales. This is also confirmed in figure 5, where a larger amount of kinetic energy is found at large scales. For large Ro, the difference between PEs and BQs is relatively small. In particular, at $R o=0.2$, the hydrostatic approximation leads to an increase of dissipation of roughly $30 \%$. On the other hand, larger differences, of about one order of magnitude, are found at smaller Ro. However, it is worth pointing out that $F r$ was kept constant at 0.01 in the aBQ runs. When $R o \sim F r$, the PEs are not expected to be a good approximation of the dynamics, as seen from (2.5b) and (2.5c).

The convergence of the BQs to the PEs for $\mathrm{Fr}$ approaching zero is illustrated in figure $10(b)$ where the energy flux for the bBQ runs is shown. The Rossby number is kept fix to 0.1 , whereas $F r$ is varied from 0.001 to 0.1 . The PE limit is plotted as well, for reference. The fact that an increased degree of stratification leads to a larger energy flux towards small scales seems inconsistent with the idea that stratification suppresses the vertical velocity and increases the two-dimensionality of the system, which according to several studies (e.g. Lilly 1983) should lead to an inverse cascade of energy. However, this result is in agreement with a number of recent studies on stratified turbulence (Riley \& deBruynKops 2003; Lindborg 2006; Brethouwer et al. 2007), showing that stratification favours a downscale energy cascade. It should be 

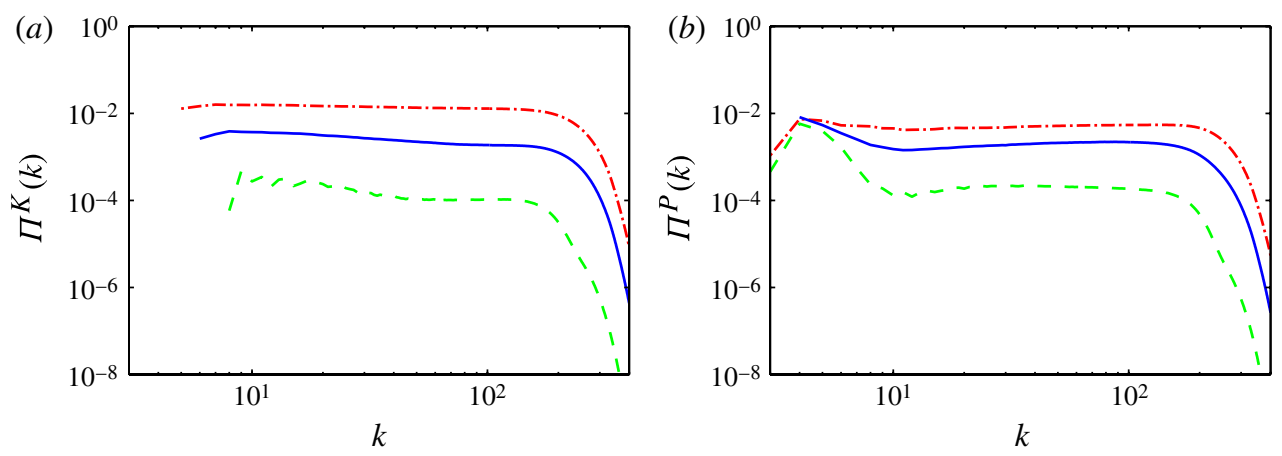

FIGURE 12. (Colour online) ( $a$ ) Kinetic and $(b)$ potential energy flux function of the wavenumber $k$ for aBQ runs:.,$-- R o=0.2 ; \_, R o=0.1 ;---, R o=0.05$.
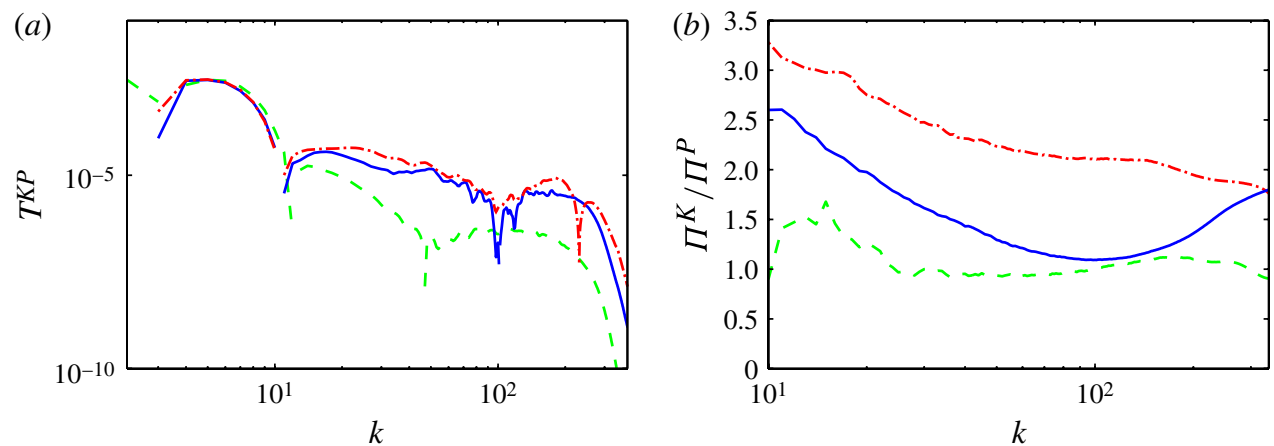

FIGURE 13. (a) Kinetic to potential buoyancy energy transfer given by (5.4). For the positive part: red curve, $R o=0.2$; blue curve, $R o=0.1$; green curve, $R o=0.05$. Negative part in dashed lines for all the curves. $(b)$ Ratio between kinetic and potential energy fluxes for the $\mathrm{aBQ}$ set: red curve, $R o=0.2$; blue curve, $R o=0.1$; green curve, $R o=0.05$

noted, however, that the Froude number dependence of the energy flux is weak as compared with the Rossby number dependence, as seen in figure 10.

Figure 12 shows the spectral flux of potential and kinetic energy, respectively, for the $\mathrm{aBQ} x$ runs. The fact that the total energy fluxes exhibit a constant range indicates that large-scale dissipation and small-scale dissipation are negligible at these scales. Indeed, large-scale dissipation is found to be two or three orders of magnitude smaller than the downscale energy transfer $\varepsilon$. Note that separate fluxes do not attain constant values to the same degree as the total flux shown in figure 10. This implies that there is a kinetic to potential energy transfer. In figure 13(a), the transfer of energy from kinetic to potential energy

$$
T^{K P}(k)=\sum_{|\boldsymbol{k}|=k} \operatorname{Re}\left(\widehat{w} \widehat{b}^{*}\right)
$$

is shown. At large scales there is a net transfer from potential to kinetic energy, as is also seen in figure 12. This is consistent with atmospheric dynamics where energy at large scales is fed by the baroclinic instability which converts potential energy to kinetic energy. Interestingly, this transfer does not depend on Ro. However, at 

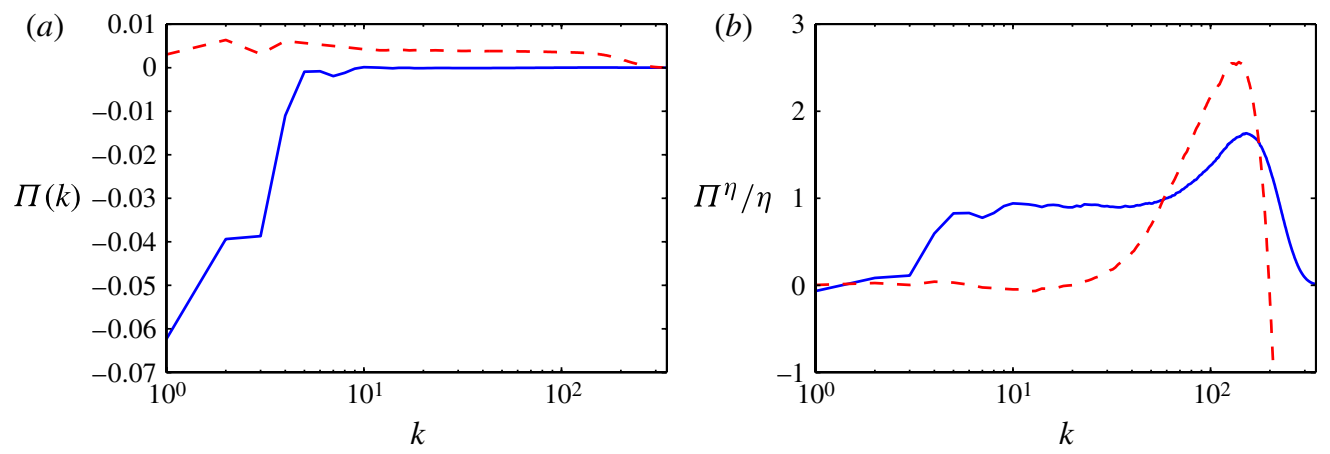

FIGURE 14. (Colour online) (a) Geostrophic energy flux $\Pi_{G}$ (-) and its complement to the total energy flux $\Pi-\Pi_{G}(----)$ for the bBQ1 run. $(b)$ Geostrophic enstrophy flux $\Pi_{G}^{\eta}$ (_- ) and its complement to the total enstrophy flux $\Pi^{\eta}-\Pi_{G}^{\eta}(---)$ for the bBQ1 run.

smaller scales, the transfer changes sign (dotted lines being the negative part) which means that there is a net transfer from kinetic energy to potential energy. This is also seen in figure $12(b)$, where, within the turbulent forward cascade, $\Pi^{P}$ is seen to slightly increase. This result is in agreement with the numerical simulations of Molemaker et al. (2010). Figure 12 indicates that the ratio between kinetic energy and potential energy fluxes varies with $R o$. We investigate this in figure $13(b)$, where the ratio $\Pi^{K} / \Pi^{P}$ is shown for the aBQ set. The ratio is not constant and increases with Ro. Moreover, due to the small but consistent transfer of energy from kinetic to potential energy, this quantity is expected to slightly decrease in the cascade. It could be speculated that at small $R o$ large part of the kinetic energy undergoes an inverse cascade and, as an effect, reduces the amount of downscale kinetic energy. At $R o=0.05$, the downscale cascade of total energy is roughly equipartitioned between kinetic and potential energy.

In order to better understand the role of ageostrophic motions, we separate the contribution of purely geostrophic motions from the total energy flux. In figure 14(a), the geostrophic energy flux is shown together with its complement to the total flux, $\Pi-\Pi_{G}$. The geostrophic energy flux attains negative values over the whole range, with large contributions only at large scales. Here, it accounts for almost the entire flux, with its complement being one order of magnitude smaller and positive. Clearly, geostrophic interactions support the inverse cascade, whereas ageostrophic motions allow for a drain of energy downscale. At wavenumbers larger than 10, the geostrophic flux becomes negligible and its complement $\Pi-\Pi_{G}$ accounts for the entire downscale energy transfer. A further decomposition of the complement energy flux to the geostrophic energy flux, $\Pi-\Pi_{G}$, shows that its dominant contribution is from the interactions between two ageostrophic modes and one geostrophic mode.

Similar conclusions apply also to the potential enstrophy fluxes which are shown in figure 14(b). In QG turbulence, potential enstrophy is a conserved quantity. Unlike energy, it cascades downscale and is finally dissipated at small scales where viscosity dominates. However, within the framework of the PEs and the BQs it is not a conserved quantity and therefore its flux, $\Pi^{\eta}$, does not generally go to zero as $k \rightarrow \infty$. Nevertheless, the geostrophic counterpart of the potential enstrophy flux, $\Pi_{G}^{\eta}$, goes to zero, as shown by the conservation relations (2.19). At large scales, the geostrophic potential enstrophy flux is the dominant contribution and attains a value of the order 


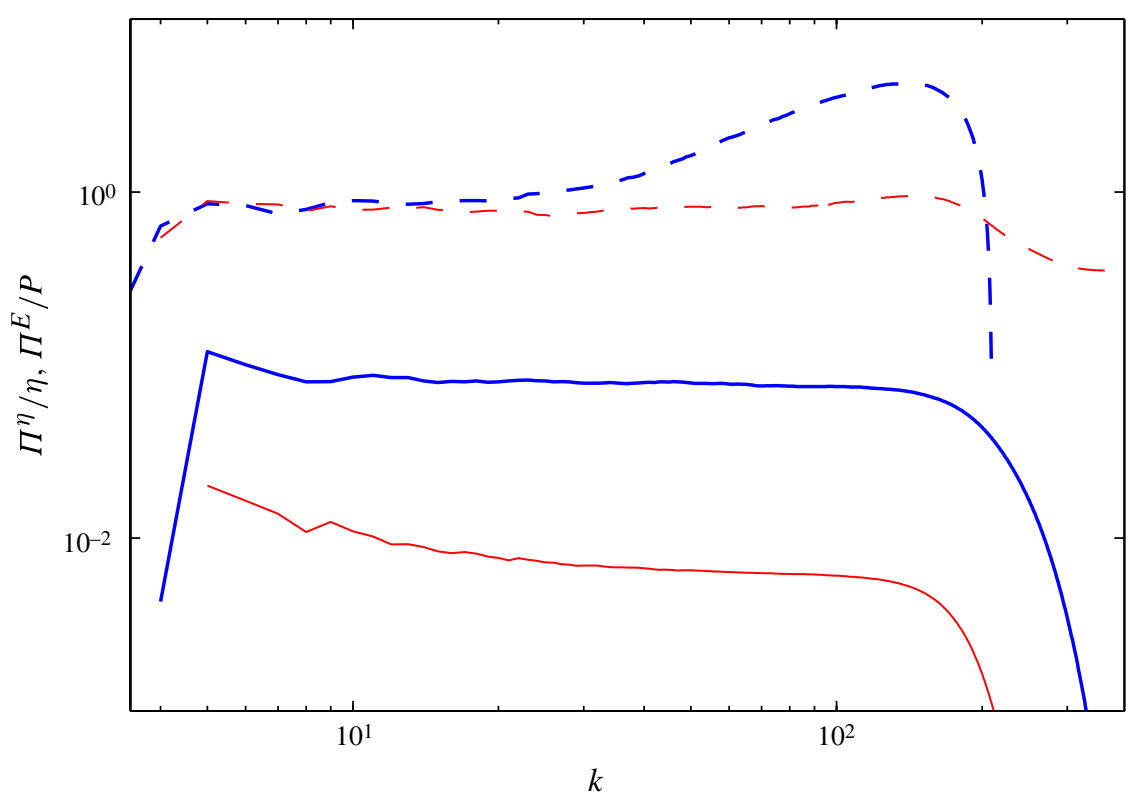

FIGURE 15. (Colour online) Energy (- - ) and enstrophy (----) fluxes scaled with the enstrophy and energy injection rates as a function of the wavenumber $k$ for the aBQ runs with $R o=0.1$ (thick lines, shown in blue online) and $R o=0.05$ (thin lines, shown in red online). Constant enstrophy and energy fluxes can be observed over the same range of wavenumbers.

of unity, showing that the injected enstrophy cascades downscale. The complement to the total potential enstrophy flux is two orders of magnitude smaller in this range, but increases with wavenumbers. At the transition wavenumber, it attains values as large as the geostrophic part. At these scales, departures from the QG prediction can be observed also for the geostrophic counterpart which shows a small bump located at large wavenumbers. Nevertheless, its magnitude stays on the order of unity.

A major finding of this study is that at non-zero but small $\mathrm{Ro}$ and $\mathrm{Fr}$ the forward enstrophy cascade and the forward energy cascade may coexist in the same range of scales. This is clearly shown in figure 15 where the total potential enstrophy flux (dashed lines) and the total energy flux (solid lines) are shown together for the $\mathrm{aBQ} 05$ and aBQ1 runs. This is remarkably true for the lower $R o$ where both fluxes attain constant values for the whole span of scales. Nevertheless, whereas all of the enstrophy cascades downscale, only a small portion of energy cascades towards small scales, the rest being transferred upscale in the inverse energy cascade. It is worth noting that despite the fact that there is a reasonably clean enstrophy downscale cascade, energy spectra deviates from the QG limit, as shown by figure 4.

\section{Wave motions}

We now investigate the role of inertia-gravity waves in the dynamics. Owing to the singularity which is present for barotropic modes in the PEs, only runs from the BQs will be considered in the following. It should be pointed out that the random forcing we introduce in the flow excites gravity waves, since all of the frequencies are forced. The waves are, however, not directly forced since the forcing is only applied to the $q$ 

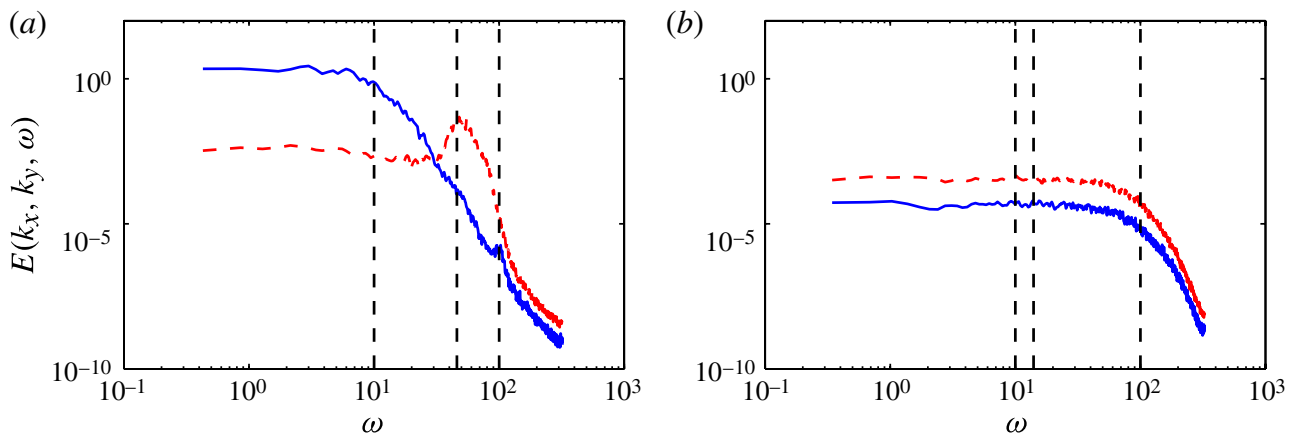

FIGURE 16. (Colour online) Examples of the time-frequency spectrum for $(a)$ a large-scale $\left(k_{h}=10, k_{v}=2\right)$ and $(b)$ a small-scale $\left(k_{h}=50, k_{v}=50\right)$ Fourier mode. - , geostrophic spectrum $E_{G} ;----$, ageostrophic spectrum $E_{A}$. Vertical helping dashed lines represent the inertial frequency $f$, the dispersion relation frequency $\omega_{d}$ and the Brunt-Väisälä frequency $N$ (from left to right).

equation. The excitement of gravity waves at the forcing scale is crucial in order to be able to investigate the possible role of gravity waves in the downscale energy cascade.

Wave motions are studied through frequency analyses. Owing to storage limitations together with the high resolutions employed, time series of only a limited number of spectral components were collected. A logarithmically spaced span of 40 wavenumbers between 1 and 330 were considered both in the horizontal and in the vertical directions. In each horizontal circle, 15 equally spaced wavenumbers were collected. Frequency spectra were computed from time series collected from each individual mode and the spectra were averaged over the horizontal circles. From (2.10), it is easy to see that wave frequencies can just lie between $\mathrm{Ro}^{-1}$ and $\mathrm{Fr}^{-1}$. Therefore, if one aims at resolving all of the possible waves in time, time resolutions of at least $\pi \mathrm{Fr}$ as well as time spans of $4 \pi R o$ are required. As the separation between $R o$ and $F r$ increases, this poses severe requirements on storage capabilities.

Equation (2.13) shows that the total energy can be separated into two components: $E_{G}$, the geostrophic part which is only associated with $q$; and $E_{A}$, the ageostrophic part which is only associated with the ageostrophic components $a_{1}$ and $a_{2}$. In particular, no mixed geostrophic/ageostrophic terms, products of $q$ and $a_{i}$, appear in the expression of the total energy. Waves are periodic motions, with $\omega=\omega_{d}$, which only pertain to $a_{1}$ and $a_{2}$. Therefore, their signatures, if present, are expected to be observed only in $E_{A}$. Nevertheless, ageostrophic components may also include other type of motions, e.g. turbulent dynamics and/or balanced ageostrophic dynamics. Wave activity is particularly intense in two regions: barotropic modes and shear modes, corresponding to pure gravity waves and pure inertial waves, respectively. As $F r$ is increased from 0.001 to 0.1 , the importance of gravity waves on the overall ageostrophic spectrum becomes smaller. Moreover, also the range of wavenumbers largely affected decreases as $\mathrm{Fr}$ becomes comparable to $R o$. On the other hand, the extent of the region pertaining to inertial waves is not very affected by changes in $F r$. It is worth noticing that the region where wave motions are most important does not coincide with the forcing wavenumbers.

We start by analysing frequency energy spectra of single Fourier coefficients in order to look for possible signature of gravity waves. In figure 16 the frequency energy spectra for two particular modes, a large-scale mode, $\left(k_{h}, k_{z}\right)=(10,2)$, and a 
small-scale mode, $\left(k_{h}, k_{z}\right)=(50,50)$, are shown for $R o=0.1$ and $F r=0.01$. In the large-scale mode, geostrophic energy dominates at low frequencies, attaining values that are about two orders of magnitude larger than the ageostrophic counterpart. At the inertial frequency, the geostrophic spectrum starts to decay, whereas the ageostrophic spectrum stays rather flat and peaks in a range between $\mathrm{Ro}^{-1}$ and $\mathrm{Fr}^{-1}$. The distinct peak corresponds to motions with a particular frequency, i.e. waves, which closely match with the frequency $\omega_{d}$ of the dispersion relation (2.10). For this frequency, ageostrophic energy dominates over the geostrophic energy, thanks to the contribution of the wave motions. When we turn to the small-scale mode, we note that no distinct peak can be observed and both the geostrophic and the ageostrophic spectra show a rather flat behaviour with comparable magnitude, indicating the absence of noticeable wave motions at this scale.

In order to give a more quantitative estimate of the role of inertia-gravity waves, we now consider spectra averaged over several modes. According to (2.10), the dispersion frequency $\omega_{d}$ is constant along straight lines in a $k_{h}-k_{z}$ plane. In order to investigate whether waves with a particular frequency can be observed, the geostrophic energy spectrum, $E_{G}\left(k_{h}, k_{z}, \omega\right)$, and the ageostrophic energy spectrum, $E_{A}\left(k_{h}, k_{z}, \omega\right)$, have been averaged over modes that have similar $\omega_{d}$. Seven frequency bands were chosen, logarithmically ranging from $R o^{-1}$ up to $F r^{-1}$ : $\omega_{\text {band }}=[10,12,18,27,39,57,84,100]$. In order to separate contributions from large-scale and small-scale motions, the averaged spectra were divided into large-scale spectra, with $k=\sqrt{k_{h}^{2}+k_{z}^{2}}<10$, and small-scale spectra, with $k>10$. In figure 17 the averaged spectra are shown. The large-scale geostrophic part shows a rather flat behaviour at high frequencies. This is clearly due to the forcing that is prescribed to be white noise in time, i.e. all of the frequencies are excited. Notwithstanding, the small-scale geostrophic spectra (shown in figure 17b) do not conserve the memory of the forcing and large decaying rates are found at high frequencies, with $E_{G}(\omega) \approx \omega^{-4}$. The cut-off frequency at which the small-scale spectrum starts to decay is of the order of the rotation rate $f$, showing that most of the energy is concentrated at frequencies smaller than or comparable to $f$.

In figure 17(c), the large-scale ageostrophic frequency spectra are plotted. The distinct peaks at $\omega=\omega_{d}$ show that wave activity is important in this range of wavenumbers. Note that both the geostrophic and ageostrophic large-scale spectra show a small but distinct peak at $\omega_{d}=\mathrm{Fr}^{-1}$. This is a spurious effect of an accumulation of energy in the barotropic mode, $k_{z}=0$, which leads to the formations of a strong wave signature at $\omega_{d}\left(k_{z}=0\right)=F^{-1}$, contaminating all modes through nonlinear interactions, both in the geostrophic and ageostrophic part. Nevertheless, for the collected modes, the amount of energy around $\omega=\mathrm{Fr}^{-1}$ is very small, around $10^{-6}$ times smaller than the total. We therefore conclude that these motions are not dynamically important. When we turn to the small-scale ageostrophic spectra in figure $17(d)$, distinct peaks cannot be observed. Instead, spectra show a rather flat behaviour on a relatively large range of frequencies. Our general conclusion is thus that wave activity is important at large scales, corresponding to wavenumbers close to the forcing scale, but is negligible at the small scale.

\section{Triad interactions}

We start by analysing the exchange of energy between geostrophic and ageostrophic modes. The quantities in the following were computed from individual flow fields and then averaged individual realizations of the aBQ set. In figure $18(a, b)$, the transfer into 
(a)

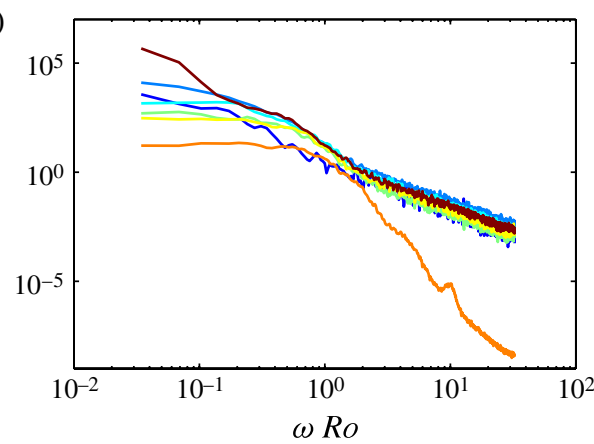

(c)

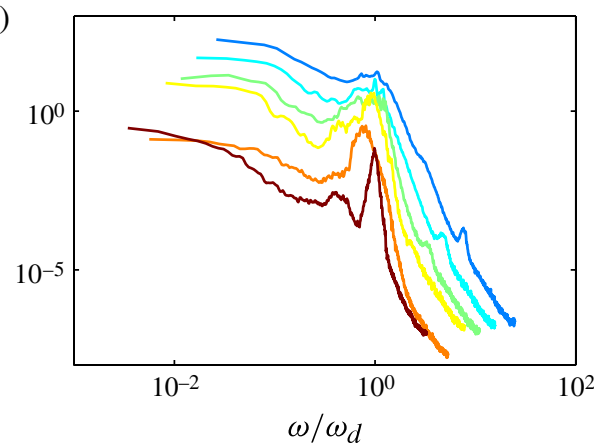

(b)

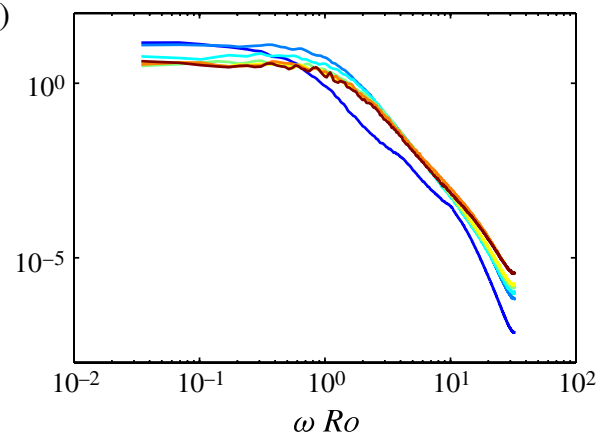

$(d)$

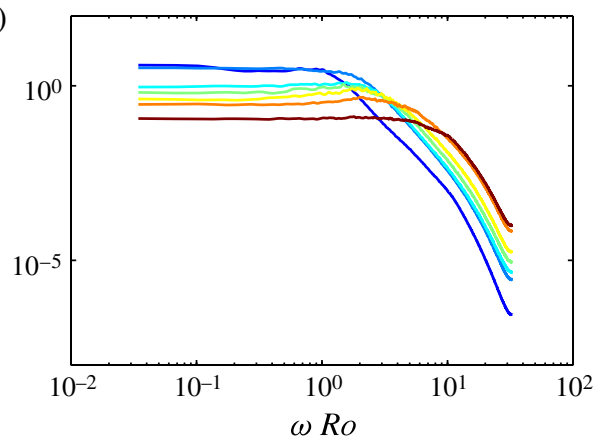

FIGURE 17. $(a, b)$ Averaged time frequency geostrophic power energy spectra. (c,d) Averaged time frequency ageostrophic power energy spectra. Parts $(a, c)$ refer to the largescales modes, whereas $(b, d)$ refer to the small-scale modes. Note that in $(c)$, the frequency axis has been rescaled with the dispersion relation frequency $\omega_{d}$, which differs from curve to curve, in order to highlight peaks due to wave motions. The data pertain to the aBQ1 run.

geostrophic energy separated in its three different contributions, as given by (2.17), is shown. Despite the averaging, curves still show a somewhat spiky behaviour. Low-pass filtered counterparts characterized by smoother trends are also shown in thicker lines. At large scales, the contribution from $T_{G G G}$ dominates, leading to an upscale cascade of energy. At smaller scales, the three parts attain comparable magnitudes with $T_{G G G}$ being preferentially negative and $T_{G A A}$ preferentially positive.

On the other hand, the large-scale transfer of energy into ageostrophic energy is mainly due to positive contributions of $T_{A G G}$. This result is consistent with the statistical mechanical analysis of Bartello (1995), suggesting that this term is mainly responsible for the transfer of energy from geostrophic motions to ageostrophic motions in the so-called process of 'geostrophic adjustment'. In figure 20, the transfer of energy into the ageostrophic motions due to geostrophic interactions, $T_{A G G}$, is investigated for the aBQ set. The largest contributions are found at large-scales where this term accounts for the conversion from geostrophic energy to ageostrophic energy. Its contribution varies with the $R o$, increasing with $R o$. Our analysis also shows that there is another term of comparable magnitude, namely $T_{A G A}$. It attains negative values, removing energy from the large-scales and producing a downscale transfer of energy. Note that if $q$ were a conserved quantity, the interaction term $T_{A G A}$ would only allow energy exchange between two ageostrophic modes, leaving the geostrophic mode unchanged, and the corresponding interaction term $T_{G A A}$ would therefore be zero. This is not the case in our simulations, as can be seen in figure $18(a, b)$. However, $T_{G A A}$ 

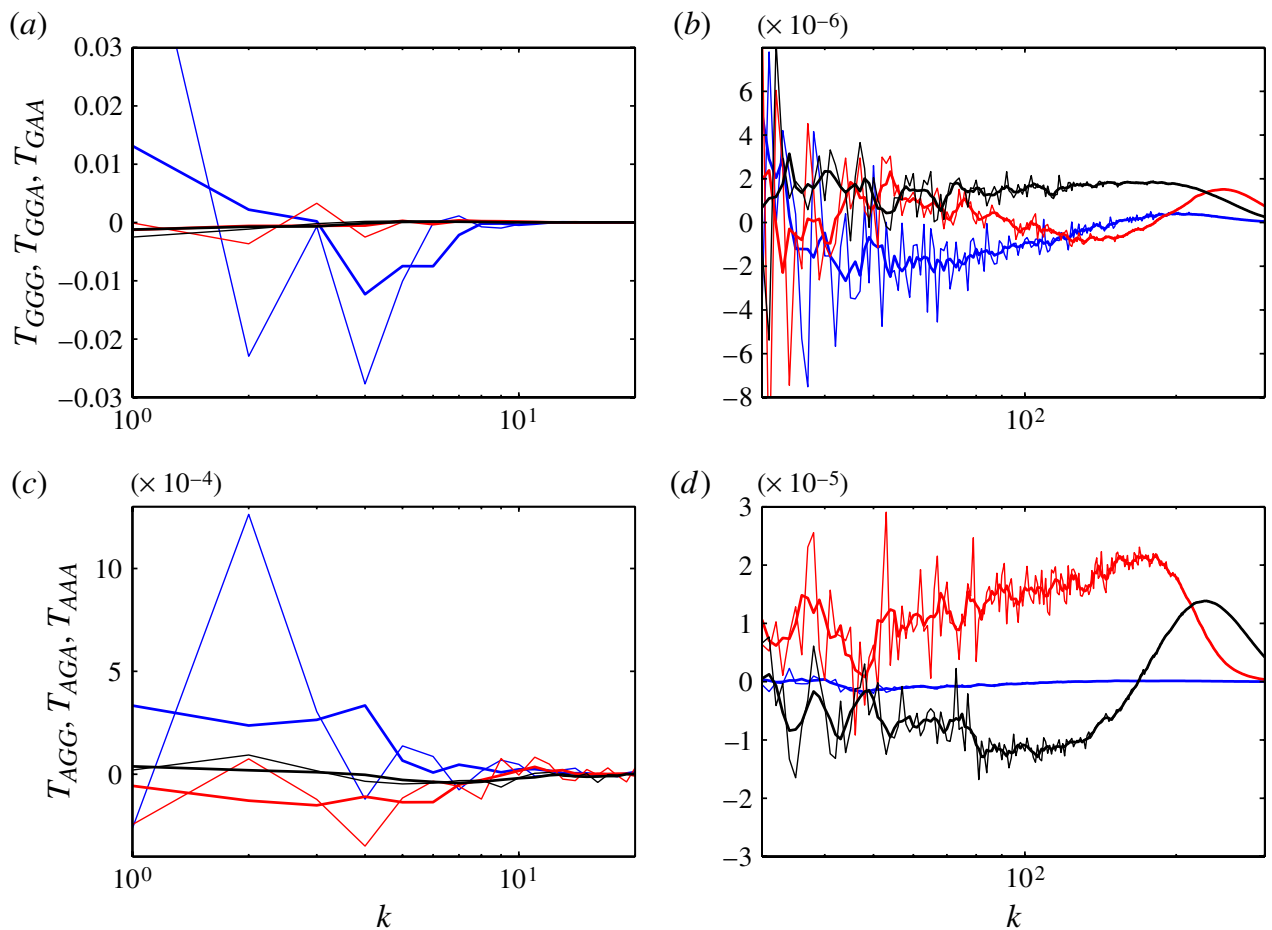

FIGURE 18. Transfer of energy into the geostrophic modes at $(a)$ large scales and $(b)$ small scales: blue curve, $T_{G G G}(\boldsymbol{k})$; red curve, $T_{G G A}(\boldsymbol{k})$; black curve, $T_{G A A}(\boldsymbol{k})$. Transfer of energy into the ageostrophic modes at $(c)$ large scales and $(d)$ small scales: blue curve, $T_{A G G}(\boldsymbol{k})$; red curve, $T_{A G A}(\boldsymbol{k})$; black curve, $T_{A A A}(\boldsymbol{k})$. Thick lines represent low-pass filtered counterparts.

is one order of magnitude smaller than $T_{A G A}$, consistent with the analysis of Bartello (1995).

The total energy transfer at small scales is dominated by two contributions, $T_{A G A}$ and $T_{A A A}$. It should be noted that the scales in figure $18(b, d)$ are different. The magnitude of the ageostrophic energy transfer terms at small scales is one order of magnitude larger than their geostrophic counterparts. In the ocean, interactions among waves are thought to have a central role in the transfer of energy among scales. How much of $T_{A G A}$ and $T_{A A A}$ is accounted for by wave dynamics is surely an interesting question. Indeed, both $T_{A G A}$ and $T_{A A A}$ present the possibility of involving resonant wave interactions. With respect to the term $T_{A G A}$, if resonance were to happen, the interaction would have to involve two waves with equal but opposite frequencies (Bartello 1995). As shown in appendix B, wave motions can be classified according to the two eigenmodes of the linear part of (2.5b) and (2.5c). Resonance must occur between one wave of the first class (pertaining to the first eigenmodes) and one of the second class of waves (pertaining to the second eigenmodes). In figure $19(c, d)$, the term $T_{A G A}$ has been further decomposed into the terms pertaining to the interaction $T_{ \pm G \pm}$ within the same class and the terms pertaining to the interaction $T_{ \pm G \mp}$ between the two different classes. Resonant wave interactions can only make contributions to $T_{ \pm G \mp}$ and not to $T_{ \pm G \pm}$. The interaction within the same class accounts for almost the whole $T_{A G A}$ term, with $T_{ \pm G \mp}$ being two orders of magnitude smaller than $T_{ \pm G \pm}$. This clearly shows that resonant wave-wave interactions cannot explain the 
(a) $\quad\left(\times 10^{-4}\right)$

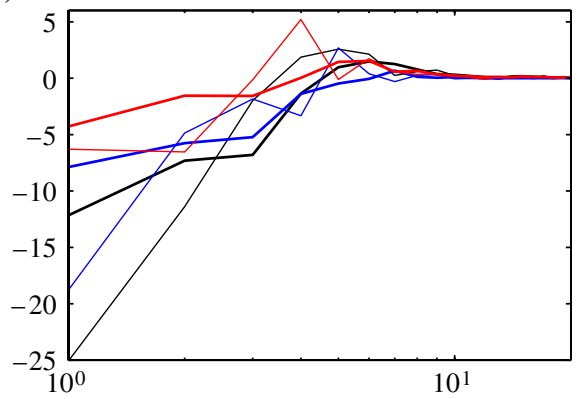

(c) $\quad\left(\times 10^{-4}\right)$

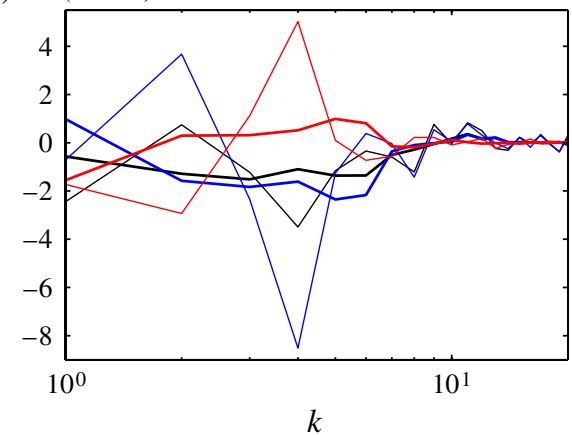

(b)

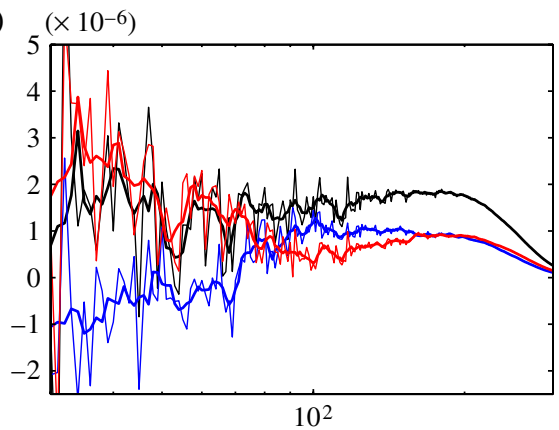

(d) $\left(\times 10^{-5}\right)$

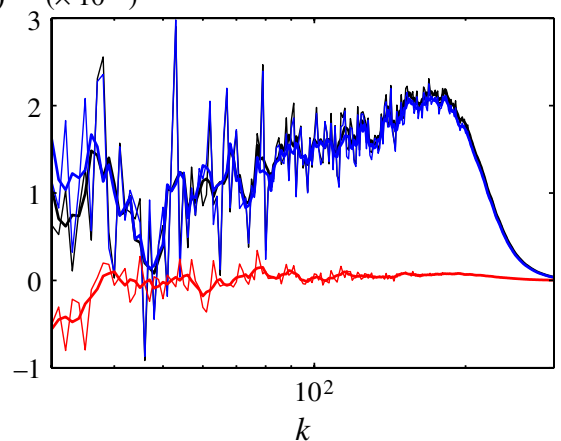

FIGURE 19. $(a, b)$ Transfer of energy into the geostrophic modes due to the interaction between one geostrophic mode and two ageostrophic modes, $(a)$ at large-scales and $(b)$ at small-scales. Black curve, total interaction $T_{G A A}(\boldsymbol{k})$; blue curve, interaction within the same class $T_{G \pm \pm}(\boldsymbol{k})$; red curve, interaction between the different class $T_{G \pm \mp}(\boldsymbol{k})$. $(c, d)$ Transfer of energy into the ageostrophic modes due to the interaction between one geostrophic mode and two ageostrophic modes, $(c)$ at large-scales and $(d)$ at small-scales. Black curve, total interaction $T_{A G A}(\boldsymbol{k})$; blue curve, interaction within the same class $T_{ \pm G \pm}(\boldsymbol{k})$; red curve, interaction between the different class $T_{ \pm G \mp}(\boldsymbol{k})$. Thick lines represent low-pass filtered counterparts.

downscale transfer of energy. At large scales, the interactions within the same class and the interaction between the classes show comparable magnitude, with the former being preferentially negative and the latter being preferentially positive. An analogous decomposition of $T_{G A A}$ is also shown in figure 19(a,b). Interactions within and between classes show similar behaviour with comparable magnitude over the whole range of scales, suggesting that there is no preferable type of interaction.

In order to further study how energy is exchanged among wavenumbers as well as the locality of the energy transfer in wavenumber space, we consider the triadic energy transfer integrated over spherical shells in wavenumber space, $T(k, p, q)$. Roughly 100 shells were chosen, logarithmically spanning the interval [1,300]. In figure 21, $T_{A G A}(k, p, q)$ is shown in a $p-q$ plane at $k=110$, i.e. within the downscale energy cascade. At this location, the effect of viscosity is negligible and the term $T_{A G A}$ constitutes the largest contribution, as shown in figure 18. As in turbulent flows, the energy transfer term $T_{A G A}$ in a $p-q$ plane concentrates in two regions, corresponding to one wavenumber of comparable magnitude with respect to $k$ and the other wavenumber being very small, i.e. strongly non-local triads. In agreement with the 


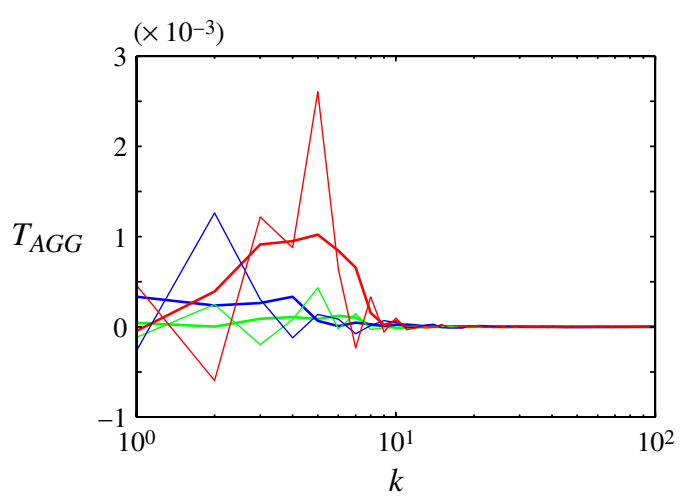

FIGURE 20. Transfer of energy into ageostrophic motions due to geostrophic interactions, $T_{A G G}$ : red curve, $R o=0.2$; blue curve, $R o=0.1$; green curve, $R o=0.05$.

(a)

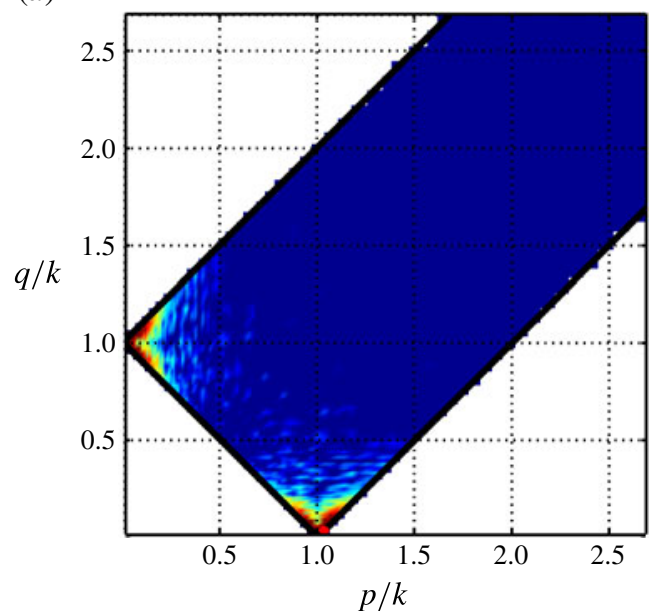

(b)

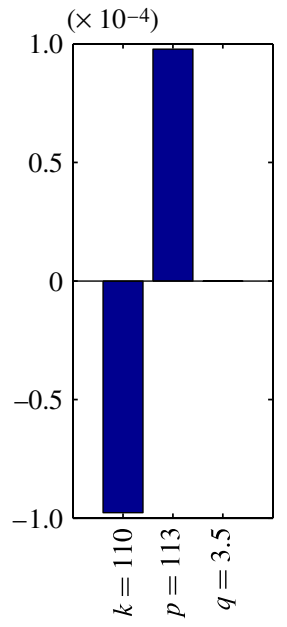

FIGURE 21. (a) Absolute value of the energy transfer function $T_{A G A}(k, p, q)$ in a $p-q$ plane at $k=110$. Note that $p$ and $q$ are rescaled with $k$. The colour axis has a logarithmic scale. $(b)$ Example of a dominant triad interaction, corresponding to the red spot in $(a)$.

results of Ohkitani \& Kida (1992) and Maltrud \& Vallis (1993), the transfer of energy, however, is between the two comparable wavenumbers, whereas the small wavenumber does not exchange energy within the triad but rather acts as a catalyser. It is worth pointing out that such triads may satisfy the resonant condition. Simple geometrical considerations reveal that the three wavenumbers involved in such triads lay along the same line in wavenumber space. From the dispersion relation (2.10), it is therefore evident that waves in these modes possess the same frequencies. However, Lelong \& Riley (1991) showed that the transfer of energy in a vortex-wave-wave interaction between the two waves tends to zero when the horizontal projection of the wave wavenumbers are parallel to each other, as in this case. Thus, also the spectral transfer of energy strongly supports the conjecture that transfer of energy between scales is a result of turbulent dynamics rather than wave dynamics. 
(a)

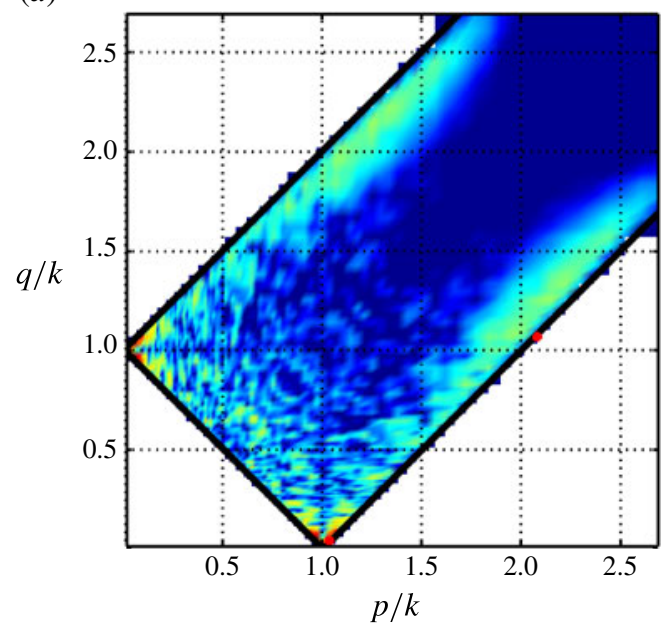

(b)

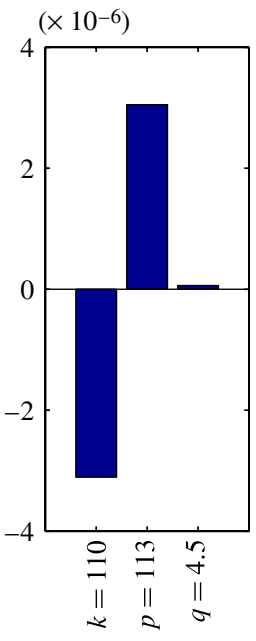

(c)

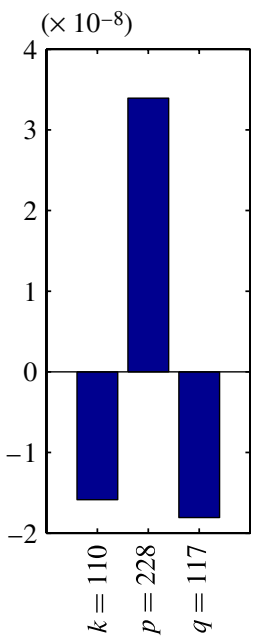

FIGURE 22. (a) Absolute value of the energy transfer function $T_{A A A}(k, p, q)$ in a $p-q$ plane at $k=110$. Note that $p$ and $q$ are rescaled with $k$. The colour axis has a logarithmic scale. $(b, c)$ Examples of two dominant triad interactions corresponding to the red spots in $(a)$.

We now turn to the transfer of energy at small scales among ageostrophic modes represented by $T_{A A A}$. Despite being smaller in magnitude, this term is of leading order at very small scales, as shown by figure 18. According to (2.19), such a term is conservative and thus only moves energy among scales. Its role mainly consists of extracting energy from the middle range of wavenumbers to feed the dissipation range, in agreement with the finding of Waite \& Bartello (2004). In order to obtain some insight in the dynamics of such a process, we investigate the quantity $T_{A A A}(k, p, q)$ in figure 22. As before, the $p-q$ plane at $k=110$ is displayed. Triad interactions show a somewhat more sparse behaviour as compared with figure 21 . Also in this case, regions pertaining to one large wavenumber (comparable to $k$ ) and one small wavenumber show intense transfer which is local and mainly between the two large wavenumbers of the triad. Such transfer is downscale and consistent with a turbulent dynamics. In addition, large energy transfers are also obtained in regions corresponding to interactions with wavenumbers larger than $k$, which lead to the net transfer of energy towards large wavenumbers seen in figure 18. Interestingly, most of the energy transfers is found in regions for which $p \approx k+q$ or $q \approx k+p$, i.e. nearly parallel vectors in a wavenumber space. Figure 22 shows the transfer of energy in such a triad. As opposed to a typical turbulent cascade, transfer of energy is highly non-local, with energy flowing out of the two smaller wavenumbers and into the largest wavenumber. Since the wavenumber vectors are aligned, waves possess all similar frequencies. The resonance condition is therefore hardly satisfied, suggesting that wave-wave-wave resonant interactions are of minor importance also in this type of energy transfer.

\section{Conclusions}

We have studied the route to dissipation in strongly stratified and rotating flows, covering a range of values of $R o$ and $F r$, representative for large-scale atmospheric flows. As expected, the BQs and PEs show similar dynamics when $F^{2} / \operatorname{Ro}^{2} \ll 1$, 
although some interesting differences also are observed. In agreement with the simulations of Vallgren et al. (2011), finite rotation rates led to departures from the QG dynamics. As Ro is increased, the amount of energy cascading upscale decreases and a fraction of the injected energy starts to cascade downscale. Thus, a non-zero Rossby number may reconcile the apparent paradox that energy dissipation should be absent in flows conforming to QG dynamics to lowest order. However, whether the ageostrophic downscale cascade of energy or other possible pathways to dissipation (as solid boundaries, wave cascade, wave breaking, etc.) are those that dominate geophysical flows is a question yet to be answered. Interestingly, the forward energy cascade towards small scales leads to a shallowing of the energy spectra, from $\sim k_{h}^{-3}$ to $\sim k_{h}^{-5 / 3}$, in agreement with the observations of Nastrom \& Gage (1985). Following Vallgren et al. (2011), the large-scale part of the spectrum can be described as the enstrophy range of a QG cascade, $E(k) \sim \eta^{2 / 3} k^{-3}$. On the other hand, at smaller scales the spectrum is of the form $E(k) \sim \varepsilon^{2 / 3} k^{-5 / 3}$ where $\varepsilon$ is the small-scale energy dissipation. Simple algebra shows that the transition wavenumber can be estimated as $k_{t} \sim \sqrt{\eta / \varepsilon}$. A model of how $k_{t}$ varies with $R o$ can be obtained if one assumes that the ratio between the small-scale dissipation and the energy injection varies as $\varepsilon / P \sim R o^{n}$. Figure 11 indicated that $n \sim 3 / 2$ for PEs and $n \sim 2$ for BQs. The enstrophy injection rate and the energy injection rate are related as $\eta \sim P k_{f}^{2}$, where $k_{f}$ is the forcing wavenumber. By combining these relations we find that the transition wavenumber varies as $k_{t} \sim k_{f} R o^{-n / 2}$. Our results are in general agreement with this estimate. For $R o=0.1$, Vallgren et al. (2011) estimated the transition scale in their simulations of the PEs at $\sim 700 \mathrm{~km}$. By applying similar arguments, a transition scale at $\sim 300 \mathrm{~km}$ is found when non-zero $F r_{h}$ is considered (aBQ1). On the other hand, the vertical energy spectra are somewhat more obscure and show a strong non-monotonic behaviour. Both in the QG and in the strongly stratified limit, a $k^{-3}$ dependence is recovered. However, for intermediate $R o$, spectra show a $k^{-5 / 3}$ range of scales. At large scales, the rotational spectrum scales as $\sim k_{h}^{-3}$, in agreement with QG dynamics, whereas the divergent spectrum is several orders of magnitude smaller and is somewhat more shallow than $k_{h}^{-5 / 3}$. At the wavenumber where the magnitudes of the rotational and divergent spectra become comparable, the rotational spectrum shallows and both spectra approximately scale as $k_{h}^{-5 / 3}$.

Spectral fluxes indicate the existence of an inertial range of scale where the energy is inviscidly transferred from the large scales to the very smallest scales. The amount of energy cascading downscale strongly increases with Ro for both BQs and PEs and weakly decreases with $\mathrm{Fr}$. For a fixed $R o$ the forward cascade is therefore strongest in the PEs, where $F r=0$. Most general circulation models use the PEs and they should therefore be able to reproduce a forward energy cascade if a sufficiently high resolution is used. This is consistent with the hypothesis that strong rotation leads to an inverse energy cascade, whereas strong stratification favours a forward energy cascade. The PE set therefore represents the limiting case for strongly stratified flows, for which the related small-scale dissipation is the upper limit. The separate kinetic and potential energy fluxes show that there is a transfer of energy from potential to kinetic energy at large scales and a kinetic to potential energy transfer at smaller scales where the energy cascade is dominant. 
Despite the fact that potential enstrophy is not a conserved quantity in PEs and $\mathrm{BQs}$, intermediate Rossby number simulations show a range of scales in which both the enstrophy and the energy flux are constant. A forward cascade of energy and potential enstrophy coexist. For $R o=0.05$ and $F r=0.01$, such a range extends over a decade. Enstrophy cascades downscale by triad interactions involving three geostrophic modes while energy is cascading downscale by interactions involving at least one ageostrophic mode, with a dominant contribution from interactions involving two ageostrophic and one geostrophic mode.

Structures characteristic of QG dynamics as filamentation and large-scale baroclinic vortices are observed in the flow. However, small-scale turbulent patches can also be found where the dissipation of energy is particularly intense. The local Froude number in the turbulent patches is of the order of unity, suggesting that $\mathrm{KH}$ instability is a potentially important mechanism supporting a direct energy cascade.

The role of internal gravity waves was investigated through time frequency analyses of time series from single Fourier modes. Frequency spectra from low wavenumber modes, $k<10$, of ageostrophic motions show distinct peaks at the characteristic wave frequency. At higher wavenumbers, $k>10$, no such peaks could be observed, indicating that waves become less important at scales where the energy cascade becomes dominant. That the downscale energy cascade is dominated by turbulent motions rather than waves is also confirmed by the investigation of triad interactions. Energy is mainly transferred by interactions between two ageostrophic and one geostrophic mode. If these interactions had been the result of resonant wave interactions the two ageostrophic modes would correspond to two waves with frequencies of equal magnitude but opposite signs. Our analysis clearly shows that this cannot be the case. The contribution to the energy transfer from interactions involving such motions is at least two orders of magnitude smaller that the total transfer. We therefore conclude that the motions of the downscale energy cascade in strongly stratified and rotating systems are genuinely turbulent.

\section{Acknowledgements}

The authors wish to thank Dr P. Augier for valuable comments on the manuscript. Computer time provided by SNIC (Swedish National Infrastructure for Computing) is gratefully acknowledged.

\section{Appendix A}

According to (2.12), the energy content in spectral space can be written in the quadratic form

$$
E(\boldsymbol{k})=\frac{1}{2}\left\{\begin{array}{l}
\hat{u}(\boldsymbol{k}) \\
\hat{v}(\boldsymbol{k}) \\
\hat{w}(\boldsymbol{k}) \\
\hat{b}(\boldsymbol{k})
\end{array}\right\}{ }^{H}\left[\begin{array}{cccc}
1 & 0 & 0 & 0 \\
0 & 1 & 0 & 0 \\
0 & 0 & F r^{2} & 0 \\
0 & 0 & 0 & 1
\end{array}\right]\left\{\begin{array}{c}
\hat{u}(\boldsymbol{k}) \\
\hat{v}(\boldsymbol{k}) \\
\hat{w}(\boldsymbol{k}) \\
\hat{b}(\boldsymbol{k})
\end{array}\right\}
$$

Here, the superscript.$^{H}$ refers to the Hermitian transpose. Using the inversion relations (2.6), we can express the primitive variable $\hat{u}(\boldsymbol{k}), \hat{v}(\boldsymbol{k}), \hat{w}(\boldsymbol{k})$ and $\hat{b}(\boldsymbol{k})$ from the 
prognostic variable $\hat{q}(\boldsymbol{k}), \hat{a}_{1}(\boldsymbol{k})$ and $\hat{a}_{2}(\boldsymbol{k})$ as

$$
\left\{\begin{array}{l}
\hat{u}(\boldsymbol{k}) \\
\hat{v}(\boldsymbol{k}) \\
\hat{w}(\boldsymbol{k}) \\
\hat{b}(\boldsymbol{k})
\end{array}\right\}=\left[\begin{array}{ccc}
\frac{i k_{y}}{k^{2}} & \frac{i R o k_{x} k_{y}}{k^{2} k_{z}} & \frac{-i R o\left(k_{x}{ }^{2}+k_{z}{ }^{2}\right)}{k^{2} k_{z}} \\
\frac{-i k_{x}}{k^{2}} & \frac{i R o\left(k_{y}{ }^{2}+k_{z}^{2}\right)}{k^{2} k_{z}} & \frac{-i R o k_{x} k_{y}}{k^{2} k_{z}} \\
0 & -\frac{i k_{y}}{k_{z}^{2}} & \frac{i k_{x}}{k_{z}^{2}} \\
\frac{k_{z}}{k^{2}} & R o \frac{k_{x}}{k^{2}} & R o \frac{k_{y}}{k^{2}}
\end{array}\right]\left\{\begin{array}{c}
\hat{q}(\boldsymbol{k}) \\
\hat{a}_{1}(\boldsymbol{k}) \\
\hat{a}_{2}(\boldsymbol{k})
\end{array}\right\} .
$$

Energy can therefore be written as

$$
E(\boldsymbol{k})=\tilde{\boldsymbol{u}}^{H} \boldsymbol{E} \tilde{\boldsymbol{u}}
$$

where $\tilde{\boldsymbol{u}}=\left\{\hat{q}, \hat{a}_{1}, \hat{a}_{2}\right\}$ and $\boldsymbol{E}$ is given by

$$
\frac{1}{2}\left[\begin{array}{ccc}
k^{-2} & 0 & 0 \\
0 & \frac{R o^{2} k_{z}^{2}\left(k_{x}^{2} k_{y}^{2}+k_{x}^{2} k_{z}^{2}+k_{y}^{4}+2 k_{y}^{2} k_{z}^{2}+k_{z}^{4}\right)+F r^{2} k^{4} k_{y}^{2}}{k^{4} k_{z}^{4}} & -\frac{k_{x} k_{y}\left(R o^{2} k_{z}^{2}+F r^{2} k^{2}\right)}{k_{z}^{4} k^{2}} \\
0 & -\frac{k_{x} k_{y}\left(R o^{2} k_{z}^{2}+F r^{2} k^{2}\right)}{k_{z}^{4} k^{2}} & \frac{R o^{2} k_{z}^{2}\left(k_{x}^{2} k_{y}^{2}+k_{y}^{2} k_{z}^{2}+k_{x}^{4}+2 k_{x}^{2} k_{z}^{2}+k_{z}^{4}\right)+F r^{2} k^{4} k_{x}^{2}}{k_{z}^{4} k^{4}}
\end{array}\right] .
$$

For barotropic modes (A 3) can still be used by replacing $\tilde{\boldsymbol{u}}$ with $\{\hat{q}, \hat{w}, \hat{b}\}$ and $\boldsymbol{E}$ with

$$
\frac{1}{2}\left[\begin{array}{ccc}
k^{-2} & 0 & 0 \\
0 & F r^{2} & 0 \\
0 & 0 & 1
\end{array}\right] .
$$

The explicit expression of $\boldsymbol{E}$ shows the decoupling between geostrophic and ageostrophic modes.

\section{Appendix B}

By retaining only the linear part, $(2.5 b)$ and $(2.5 c)$ can be written as

$$
\left[\begin{array}{cc}
k_{z}^{2}+k_{y}^{2} \frac{F r^{2}}{R o^{2}} & -\frac{F r^{2}}{R o^{2}} k_{x} k_{y} \\
-\frac{F r^{2}}{R o^{2}} k_{x} k_{y} & k_{z}^{2}+k_{x}^{2} \frac{F r^{2}}{R o^{2}}
\end{array}\right] \frac{\partial}{\partial t}\left\{\begin{array}{l}
\hat{a}_{1} \\
\hat{a}_{2}
\end{array}\right\}=\frac{1}{R o}\left[\begin{array}{cc}
-k_{x} k_{y} & k_{z}^{2}+k_{x}^{2} \\
-k_{z}^{2}-k_{y}^{2} & +k_{x} k_{y}
\end{array}\right]\left\{\begin{array}{l}
\hat{a}_{1} \\
\hat{a}_{2}
\end{array}\right\},
$$

which can be recast in an eigenvalue problem for the complex frequency $\lambda$

$$
(\boldsymbol{B}-\lambda \boldsymbol{A}) \boldsymbol{a}=0
$$

where $\boldsymbol{a}=\left[\hat{a}_{1}, \hat{a}_{2}\right]^{\mathrm{T}}$. The discriminant of (B 2) gives the dispersion relation (2.10), i.e. $\lambda_{1,2}= \pm i \omega_{d}$. The matrix $\boldsymbol{M}$ whose columns are the eigenvectors of (B 1) is a linear operator which allows to project $\boldsymbol{a}$ on the eigenvector basis

$$
a=M e \quad e=M^{-1} a
$$

where $\boldsymbol{e}$ is the projection of $\boldsymbol{a}$ in the eigenvector basis. The first component of $\boldsymbol{e}$ pertains to waves with positive frequency whereas the second component of $\boldsymbol{e}$ 
pertains to waves with negative frequencies. After projecting, the normal modes used by Bartello (1995) are recovered.

We divide the transfer term $T_{A G A}$ into two parts, $T_{ \pm G \pm}$ and $T_{ \pm G \mp}$, where the first part contains contributions involving the same eigenvectors and the second part contains contributions involving two different eigenvectors. To calculate these two parts we need to separate the ageostrophic fields into two fields associated with each of the eigenvectors. This can easily be done by projecting $a_{1}$ and $a_{2}$ on the eigenvector basis, setting either the first class or the second class of modes to zero and finally transforming back to the normal basis which the inversion relations (2.6) can be applied to.

\section{REFERENCES}

Augier, P., Chomaz, J.-M. \& Billant, P. 2012 Spectral analysis of the transition to turbulence from a dipole in stratified fluids. J. Fluid Mech. 713, 86-108.

BARTELlO, P. 1995 Geostrophic adjustment and inverse cascades in rotating stratified turbulence. J. Atmos. Sci. 52, 4410-4428.

BARTELLO, P. 2010 Quasigeostrophic and stratified turbulence in the atmosphere. In IUTAM Symposium on Turbulence in the Atmosphere and Oceans (ed. D. Dritschel), IUTAM Bookseries (closed), vol. 28, pp. 117-130. Springer.

Bellet, F., Godeferd, F. S., Scott, J. F. \& CAmbon, C. 2006 Wave turbulence in rapidly rotating flows. J. Fluid Mech. 562, 83-121.

Billant, P. \& Chomaz, J.-M. 2001 Self-similarity of strongly stratified inviscid flows. Phys. Fluids 13 (6), 1645-1651.

Boer, G. J. \& Shepherd, T. G. 1983 Large-scale two-dimensional turbulence in the atmosphere. J. Atmos. Sci. 40, 164-184.

Boffetta, G. \& Musacchio, S. 2010 Evidence for the double cascade scenario in twodimensional turbulence. Phys. Rev. E 82, 016307.

Brethouwer, G., Billant, P., Lindborg, E. \& Chomaz, J.-M. 2007 Scaling analysis and simulation of strongly stratified turbulent flows. J. Fluid Mech. 585, 343-368.

Cambon, C., Mansour, N. N. \& Godeferd, F. S. 1997 Energy transfer in rotating turbulence. J. Fluid Mech. 337, 303-332.

Canuto, C., Hussaini, M. Y., Quarteroni, A. \& ZAng, T. A. 1988 Spectral methods in Fluid Dynamics. Springer.

Charney, Jule G. 1971 Geostrophic turbulence. J. Atmos. Sci. 28 (6), 1087-1094.

Cho, J. Y. N. \& LindborG, E. 2001 Horizontal velocity structure functions in the upper troposphere and lower stratosphere 1. Observations. J. Geophys. Res. 106 (D10), 10223-10232.

FERRARI, R. \& WUNSCH, C. 2009 Ocean circulation kinetic energy: reservoirs, sources, and sinks. Annu. Rev. Fluid Mech. 41, 253-282.

Gargett, A. E., Hendricks, P. J., Sanford, T. B., Osborn, T. R. \& Williams, A. J. 1981 A composite spectrum of vertical shear in the upper ocean. J. Phys. Oceanogr. 11, 1258-1271.

Garrett, C. \& Munk, W. 1979 Internal waves in the ocean. Annu. Rev. Fluid Mech. 11, 339-369.

GiLl, A. E. 1982 Atmosphere-Ocean Dynamics. Academic.

Godeferd, F. S. \& CAMBon, C. 1994 Detailed investigation of energy transfers in homogeneous stratified turbulence. Phys. Fluids 6 (6), 2084-2100.

Hamilton, K., TAKahashi, Y. O. \& Ohfuchi, W. 2008 Mesoscale spectrum of atmospheric motions investigated in a very fine resolution global general circulation model. J. Geophys. Res. 113 (18).

Kitamura, Y. \& Matsuda, Y. 2006 The $k_{H}^{-3}$ and $k_{H}^{-5 / 3}$ energy spectra in stratified turbulence. Geophys. Res. Lett. 33, L05809. 
Kraichnan, R. H. 1967 Inertial ranges in two-dimensional turbulence. Phys. Fluids 10 (7), $1417-1423$.

Leith, C. E. \& Kraichnan, R. H. 1972 Predictability of turbulent flows. J. Atmos. Sci. 29, 1041-1058.

LELONG, M.-P. \& RILEY, J. J. 1991 Internal wave-vortical mode interactions in strongly stratified flows. J. Fluid Mech. 232, 1-19.

LILLY, D. K. 1983 Stratified turbulence and the mesoscale variability of the atmosphere. J. Atmos. Sci. 40 (3), 749-761.

LindBorg, E. 2006 The energy cascade in a strongly stratified fluid. J. Fluid Mech. 550, 207-242.

LINDBORG, E. 2007 Horizontal wavenumber spectra of vertical vorticity and horizontal divergence in the upper troposphere and lower stratosphere. J. Atmos. Sci. 64, 1017.

Lindborg, E. \& BRethouwer, G. 2007 Stratified turbulence forced in rotational and divergent modes. J. Fluid Mech. 586, 83-108.

Lvov, Y. V., Polzin, K. L. \& Yokoyama, N. 2011 Resonant and near-resonant internal wave interactions. J. Phys. Oceanogr. 42 (5), 669-691. ArXiv e-prints.

Maltrud, M. E. \& VAllis, G. K. 1993 Energy and enstrophy transfer in numerical simulations of two-dimensional turbulence. Phys. Fluids 5, 1760-1775.

McComas, C. H. \& Bretherton, F. P. 1977 Resonant interaction of oceanic internal waves. J. Geophys. Res. 82, 1397-1412.

MCWilliams, James C. 2010 A perspective on submesoscale geophysical turbulence. In IUTAM Symposium on Turbulence in the Atmosphere and Oceans (ed. D. Dritschel), IUTAM Bookseries (closed), vol. 28, pp. 131-141. Springer.

Ménesguen, C., Hua, B. L., Papenberg, C., Klaeschen, D., Géli, L. \& Hobbs, R. 2009 Effect of bandwidth on seismic imaging of rotating stratified turbulence surrounding an anticyclonic eddy from field data and numerical simulations. Geophys. Res. Lett. 360.

Molemaker, M. J. \& MCWilliams, J. C. 2010 Local balance and cross-scale flux of available potential energy. J. Fluid Mech. 645, 295-314.

Molemaker, M. J., MCWilliams, J. C. \& CAPeT, X. 2010 Balanced and unbalanced routes to dissipation in an equilibrated Eady flow. J. Fluid Mech. 654, 35-63.

Molemaker, M. J., McWilliams, J. C. \& YaVneh, I. 2005 Baroclinic instability and loss of balance. J. Phys. Ocean. 35, 1505-1517.

Muller, P., McWilliams, J. C. \& Molemaker, M. J. 2005 Routes to dissipation in the ocean: the 2D/3D turbulence conundrum. In Marine Turbulence: Theories, Observations, and Models (ed. H. Z. Baumert, J. Simpson \& J. Sündermann), pp. 397-405. Cambridge University Press.

MunK, W. 1981 Internal waves and small-scale processes. Evol. Phys. Oceanogr. 264-291.

NAStrom, G. D. \& GAGE, K. S. 1985 A climatology of atmospheric wavenumber spectra of wind and temperature observed by commercial aircraft. J. Atmos. Sci. 42, 950-960.

NikURAshin, M. \& FERRARI, R. 2011 Global energy conversion rate from geostrophic flows into internal lee waves in the deep ocean. Geophys. Res. Lett. 38, 8610.

OhKitani, K. 1990 Non-locality in a forced two-dimensional turbulence. Phys. Fluids A 2 (9), $1529-1531$.

OhkitAni, K. \& KIDA, S. 1992 Triad interactions in a forced turbulence. Phys. Fluids A 4 (4), 794-802.

Pedlosky, J. 1987 Geophysical Fluid Dynamics. Springer.

PhILlips, O. M. 1981 Wave interactions - the evolution of an idea. J. Fluid Mech. 106, 215-227.

PolzIN, K. L. \& LVOV, Y. V. 2011 Toward regional characterizations of the oceanic internal wavefield. Rev. Geophys. 49, 4003.

Riley, J. J. \& DEBRUYNKops, S. M. 2003 Dynamics of turbulence strongly influenced by buoyancy. Phys. Fluids 15 (7), 2047-2059.

SCOTT, R. K. 2007 Nonrobustness of the two-dimensional turbulent inverse cascade. Phys. Rev. E 75, 046301 . 
SKAMAROCK, W. C. 2004 Evaluating mesoscale NWP models using kinetic energy spectra. Mon. Weath. Rev. 132 (12), 3019-3032.

Staquet, C. \& Sommeria, J. 2002 Internal gravity waves: from instabilities to turbulence. Annu. Rev. Fluid Mech. 34, 559-593.

Takahashi, Y. O., Hamilton, K. \& Ohfuchi, W. 2006 Explicit global simulation of the mesoscale spectrum of atmospheric motions. Geophys. Res. Lett. 33, L12812.

VALLGREN, A. 2011 Infrared reynolds number dependency of the two-dimensional inverse energy cascade. J. Fluid Mech. 667, 463-473.

Vallgren, A., Deusebio, E. \& Lindborg, E. 2011 A possible explanation of the atmospheric kinetic and potential energy spectra. Phys. Rev. Lett. 99, 99-101.

VAllgRen, A. \& LindBorg, E. 2010 Charney isotropy and equipartition in quasi-geostrophic turbulence. J. Fluid Mech. 656, 448-457.

VAllgren, A. \& LindBorg, E. 2011 The enstrophy cascade in forced two-dimensional turbulence. J. Fluid Mech. 671, 168-183.

VAlLis, G. K. 2006 Atmospheric and Oceanic Fluid Dynamics: Fundamentals and Large-scale Circulation (electronic version). Cambridge University Press.

Viúdez, Á. \& DRITsChel, D. G. 2006 Spontaneous generation of inertiagravity wave packets by balanced geophysical flows. J. Fluid Mech. 553, 107-117.

WAite, M. L. \& BARTello, P. 2004 Stratified turbulence dominated by vortical motion. J. Fluid Mech. 517, 281-308.

Waite, M. L. \& BARTEllo, P. 2006 The transition from geostrophic to stratified turbulence. J. Fluid Mech. 568, 89-108.

WAite, M. L. \& SNYDer, C. 2009 The mesoscale kinetic energy spectrum of a baroclinic life cycle. J. Atmos. Sci. 66, 883.

WUNSCH, C. \& FERRARI, R. 2004 Vertical mixing, energy, and the general circulation of the oceans. Annu. Rev. Fluid Mech. 36, 281-314. 